\title{
B cell-intrinsic TLR9 expression is protective in murine lupus
}

\author{
Jeremy S. Tilstra, ${ }^{1}$ Shinu John, ${ }^{2}$ Rachael A. Gordon, ${ }^{3}$ Claire Leibler, ${ }^{3}$ Michael Kashgarian, ${ }^{4}$ Sheldon Bastacky, ${ }^{1}$ \\ Kevin M. Nickerson, ${ }^{2,3}$ and Mark J. Shlomchik ${ }^{2,3}$ \\ 'Department of Pathology, University of Pittsburgh School of Medicine, Pittsburgh, Pennsylvania, USA. ${ }^{2}$ Department of Laboratory Medicine, Yale University School of Medicine, New Haven, Connecticut,
}

USA. ${ }^{3}$ Department of Immunology, University of Pittsburgh School of Medicine, Pittsburgh, Pennsylvania, USA. ${ }^{4}$ Department of Pathology, Yale University School of Medicine, New Haven, Connecticut, USA.

\begin{abstract}
Toll-like receptor 9 (TLR9) is a regulator of disease pathogenesis in systemic lupus erythematosus (SLE). Why TLR9 represses disease while TLR7 and MyD88 have the opposite effect remains undefined. To begin to address this question, we created 2 alleles to manipulate TLR9 expression, allowing for either selective deletion or overexpression. We used these to test cell type-specific effects of TIr9 expression on the regulation of SLE pathogenesis. Notably, TIr9 deficiency in B cells was sufficient to exacerbate nephritis while extinguishing anti-nucleosome antibodies, whereas T/r9 deficiency in dendritic cells (DCs), plasmacytoid DCs, and neutrophils had no discernable effect on disease. Thus, B cell-specific TIr9 deficiency unlinked disease from autoantibody production. Critically, B cell-specific TIr9 overexpression resulted in ameliorated nephritis, opposite of the effect of deleting Tlr9. Our findings highlight the nonredundant role of B cell-expressed TLR9 in regulating lupus and suggest therapeutic potential in modulating and perhaps even enhancing TLR9 signals in B cells.
\end{abstract}

\section{Introduction}

Systemic lupus erythematosus (SLE) is an autoimmune disease characterized by loss of tolerance to nuclear antigens, immune cell activation, autoantibody production, and multiorgan damage. SLE is a complex disease involving the dysregulation of multiple immune cell lineages, and numerous cellular and genetic mediators of SLE pathogenesis have been elucidated. However, none have been so robustly recapitulated in multiple mouse models as components of the toll-like receptor (TLR) signaling pathways $(1,2)$. Among these, several components of the TLR signaling pathway have been identified as risk alleles in patients with SLE, including TLR7, TLR8, TLR9, IRAK1, IRAK4, OPN, and ACP1 (3-5).

TLRs are a family of conserved surface and endosomal pattern recognition receptors that can detect microbial products. Ligation of TLRs culminates in the activation of transcription factors, including NF- $\mathrm{NB}$ and interferon regulatory factors. Genetic studies in murine models of SLE highlight the endosomal TLRs TLR7, TLR8, and TLR9 as critical regulators of SLE pathogenesis $(1,2)$. TLR7 and TLR8 are cellular sensors for ssRNA, whereas TLR9 senses dsDNA $(1,6)$. We have previously shown that TLR7, TLR9, and their shared signaling adaptor MyD88 are indispensable for autoantibody production, with TLR9 driving anti-DNA antibodies (i.e., anti-double stranded DNA and anti-nucleosome) and TLR7 driving anti-RNA antibody (i.e., anti-Sm) production $(7,8)$. Both

Authorship note: JST, SJ, and RAG contributed equally to this work and are co-first authors.

Conflict of interest: The authors have declared that no conflict of interest exists. Copyright: (c) 2020, American Society for Clinical Investigation.

Submitted: August 5, 2019; Accepted: March 5, 2020; Published: May 11, 2020.

Reference information: / Clin Invest. 2020;130(6):3172-3187.

https://doi.org/10.1172/JCl132328.
anti-DNA and anti-RNA classes of autoantibody were absent in MyD88-deficient lupus-prone mice.

Genetic overexpression of Tlr7 is a driver of SLE pathogenesis and is a major contributor to disease in Y chromosome-linked autoimmune accelerator-associated ( $\mathrm{Ya}$-associated) models of disease (9). Moreover, genetic deletion of $T l r 7$ in the MRL/lpr mouse model of lupus resulted in ameliorated kidney disease and reduced immune activation (8). Strikingly, and in contrast to the prevailing hypothesis at the time, global Tlr9 deficiency instead resulted in decreased survival with exacerbated nephritis and dermatitis, despite the loss of anti-DNA and specifically anti-nucleosome autoantibodies (8). Furthermore, Tlr9-regulated disease - manifested by worse disease in its absence - is dependent on $\operatorname{Tl} 7$ (7). The protective role of Tlr9 in SLE was counterintuitive given that (a) TLRs are thought of as proinflammatory receptors, (b) TLR9 signaling is responsible for anti-DNA antibody production (8), which in turn was long thought to be a pathogenic mediator of disease, and (c) TLR9 signals similarly to TLR7, an accelerator of disease (10). Since the protective role of TLR9 in SLE was first reported, this finding has been confirmed in at least 7 models of lupus, including MRL/ lpr, MRL/+, B6/lpr, Ali5 B6, Nba2.Yaa, WASp, and pristane (11-17). In each of these models, when $\operatorname{Tl} 9$ was deleted severity of renal disease was increased. In nearly all the lupus models examined, $\operatorname{Tr} 9$ deficiency led to a loss of anti-DNA autoantibodies.

Despite substantial research, it remains unclear why TLR9 and TLR7 have paradoxical effects on SLE pathogenesis, especially as these 2 receptors are thought to engage nearly identical downstream signaling pathways (10). Insights into this issue will be important for understanding lupus pathogenesis, designing lupus therapy, and possibly for understanding lupus patient heterogeneity. Importantly, deciphering why TLR7 and TLR9 play such different roles in SLE will provide basic insights into the biology of these critical TLRs and TLR signaling in general. One hypothesis to explain the 


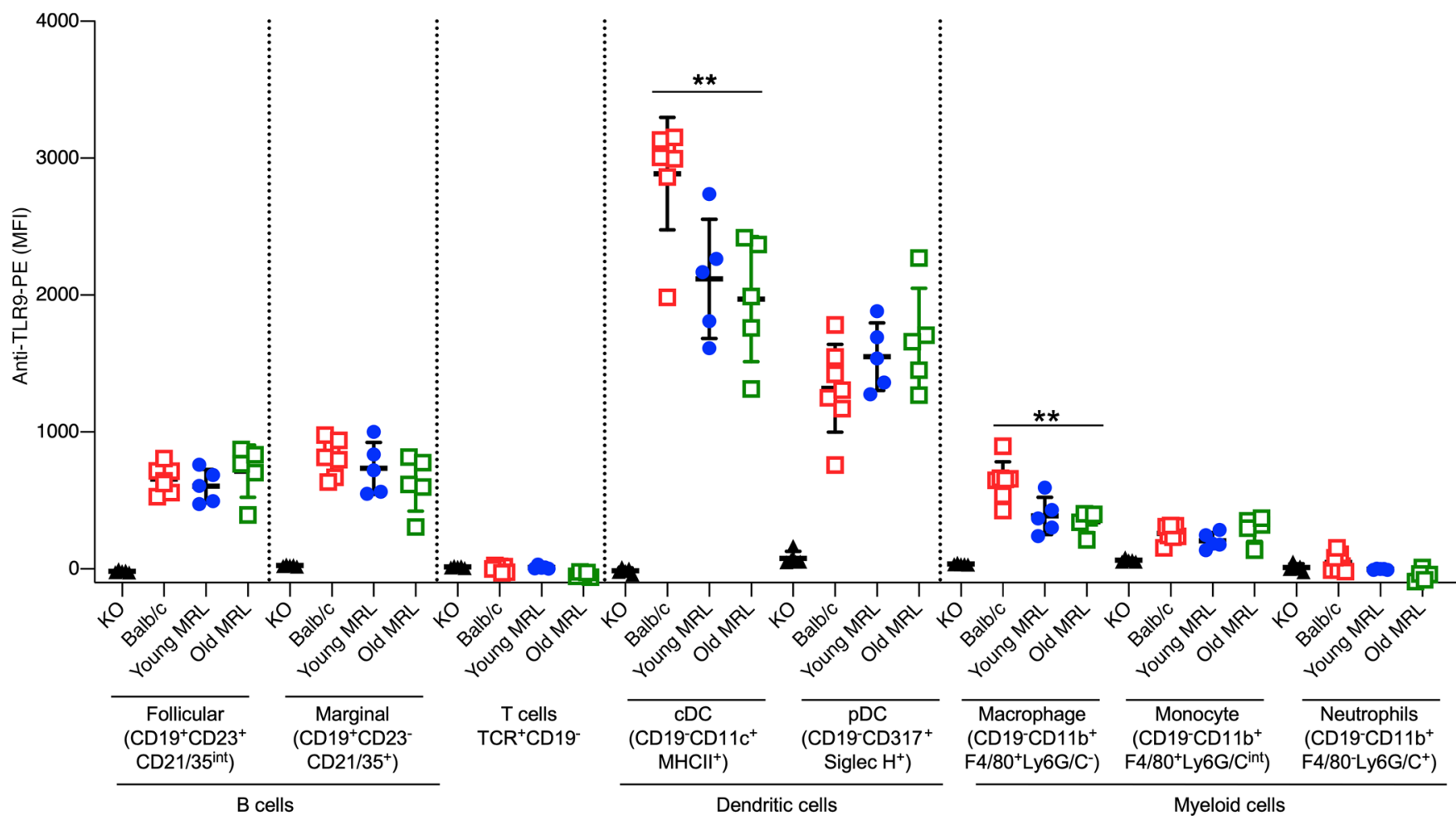

Figure 1. TLR9 is expressed on B cell, DC, and myeloid populations. Quantification of TLR9 MFI is shown for each of the defined populations representing $\mathrm{TLRg}^{-/-} \mathrm{BALB} / \mathrm{c}(n=5), \mathrm{BALB} / \mathrm{c}(n=7), 5$-week-old prediseased MRL/Ipr $(n=5)$, and diseased 21-week-old MRL//pr mice $(n=5)$. Scatter plots represent individual mice, with bars identifying mean and error bars representing standard deviations. ${ }^{*} P<0.01$, as determined by ANOVA with Tukey's multiple comparison test evaluating for differences within each cell type.

dichotomous effects of TLR7 and TLR9 is that there are cell typespecific roles for each TLR, and while TLR9 may be protective due to its effects in one cell type, TLR7 may accelerate disease due to its effects in another cell type. Alternatively, and nonexclusively, TLR9 may regulate TLR7 in a cis fashion within the same cell type by competing for shared rate-limiting downstream signaling components.

To unravel the mechanisms behind this unsolved paradox, a key step is to determine the cell-specific role of the TLRs in SLE - the goal of this study. Here, we evaluated the effects of TLR9 expression in multiple target populations to identify which, if any, regulate SLE in a TLR9-dependent fashion. This was important because multiple hematopoietic cell lineages express TLR9 and could modulate disease. These cell lineages include B cells (18$20)$, neutrophils $(21,22)$, macrophages $(23,24)$, DCs $(25,26)$, and plasmacytoid DCs (pDCs) $(26,27)$.

To address this, we generated 2 alleles to manipulate TLR9 expression, allowing either selective deletion or overexpression using different cell type-specific Cre-expressing lines. These alleles were crossed onto appropriate autoimmune-prone genetic backgrounds for this study. We evaluated the effect of TLR9 loss using a conditional Tlr9 knockout (Tlr $\left.9^{f}\right)$ in B cells (CD19-Cre), DCs (CD11c-Cre), neutrophils (MRP8-Cre), and myeloid cells, both macrophages and neutrophils (LysM-Cre). Based on the results of these studies, we determined the reciprocal effect of Tlr9 overexpression in B cells. This strategy allowed us to delineate the contributions of TLR9 signaling in various hematopoietic cell types in lupus pathogenesis. Indeed, we found striking cell type-specific dependence on Tlr9 expression for the regulation of lupus. Either over- or underexpression in B cells led to suppression or exacerbation of lupus phenotypes, respectively. Deletion of Tlr 9 via any of the other tested Cre lines failed to show a phenotype, together suggesting that B cell TLR9 expression was both necessary and sufficient to modulate SLE pathogenesis.

\section{Results}

TLR9 is expressed in B cells, myeloid cells, and DC lineages. Previously, others and we demonstrated that global Tlr9 deficiency results in exacerbated disease in murine lupus. Employing a Crelox approach, we set out to determine which hematopoietic cell type(s) mediated this acceleration/exacerbation of disease.

To date, a comprehensive analysis has not shown which cell lineages express TLR9 during autoimmunity, the process of which could alter or induce expression. Therefore, we evaluated TLR9 expression in varying immune cell subsets in nonautoimmune and lupus prone prediseased and diseased MRL/lpr mice at the protein level using a relatively new antibody reagent (28). RNAseq data compiled on ImmGen from nonautoimmune mice suggested transcription of Tlr9 in DC, B cell, and monocyte subsets, but no detectable expression on $\mathrm{T}$ cell populations (29). We extended these data at the protein level by intracellular flow cytometry and further delineated TLR9 expression on specific lymphocyte and myeloid subsets (Figure 1). As expected, BALB/c mice had TLR9 expression that correlated with the previously published transcriptional data (29). Given that TLR9 expression may have been altered in the setting of autoimmunity, we aimed to determine if the expression patterns of autoimmune MRL/lpr mice differed 
A

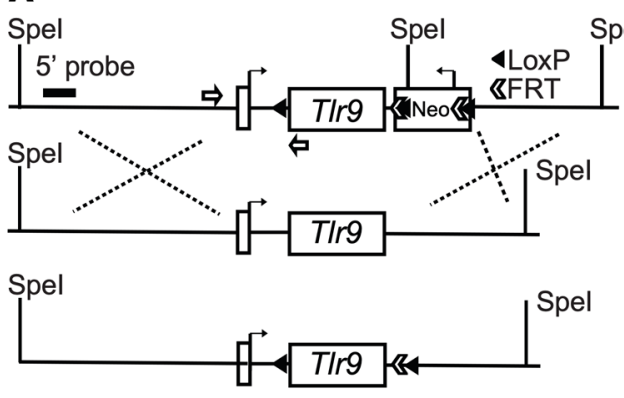

C

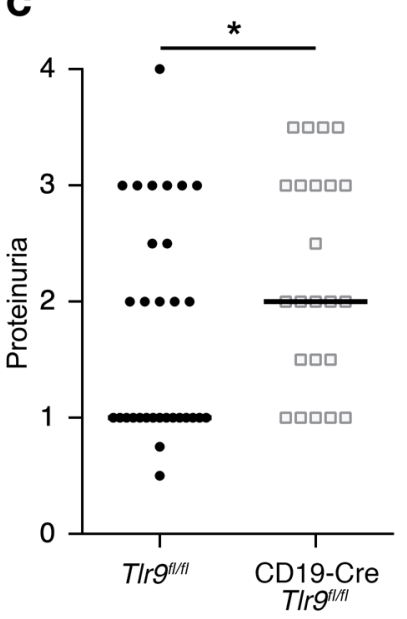

D

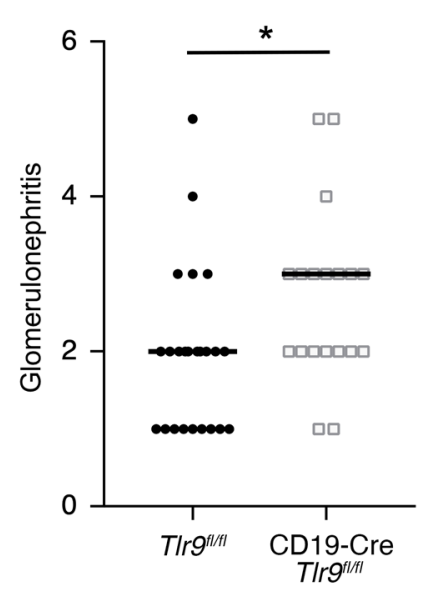

\section{B}

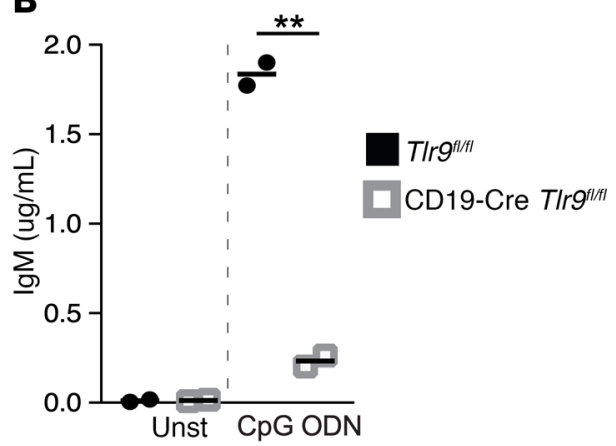

E

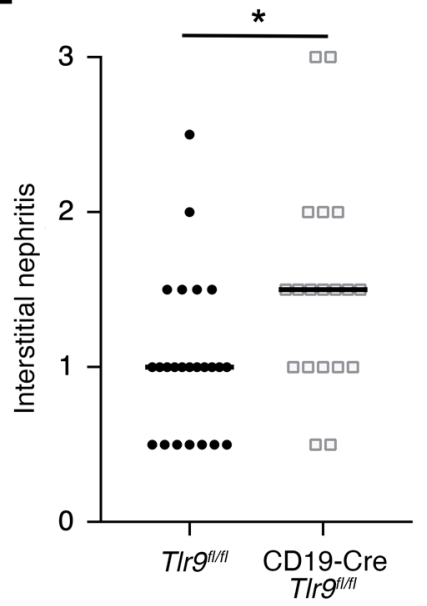

$\mathbf{F}$

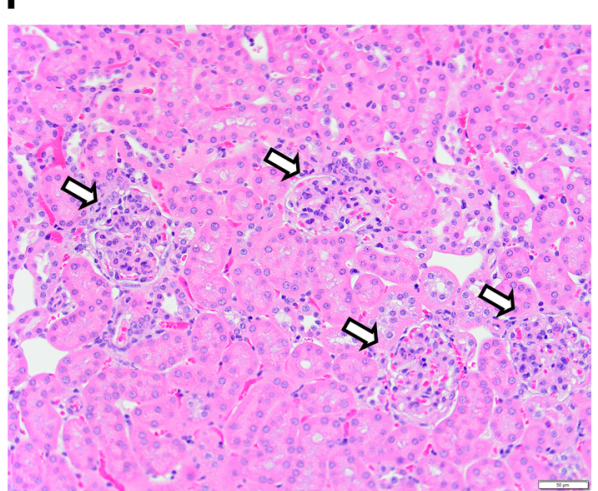

TIrg ${ }^{f / t}$

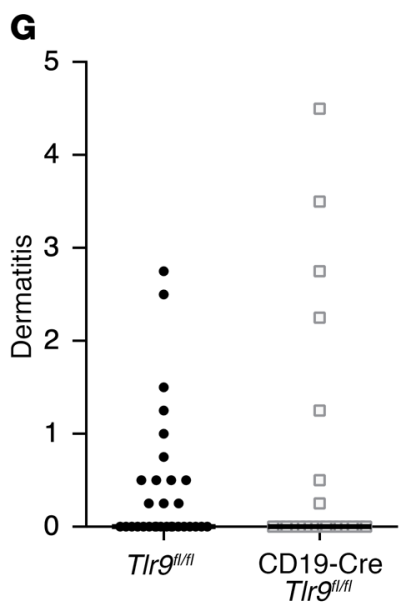

Targeting construct WT allele

TIrg"l allele after Neo deletion

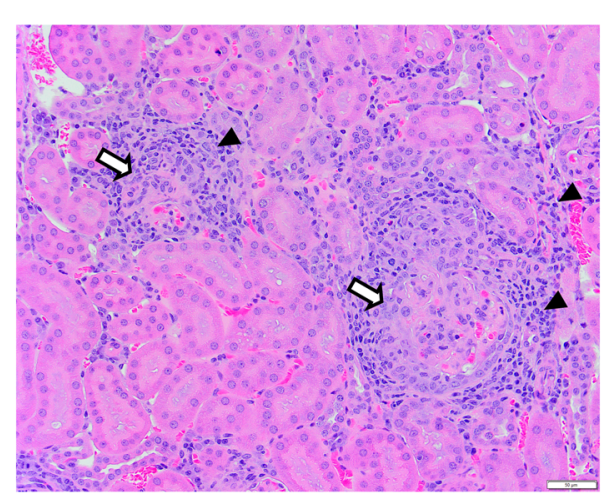

CD19-Cre T/rgt/itt
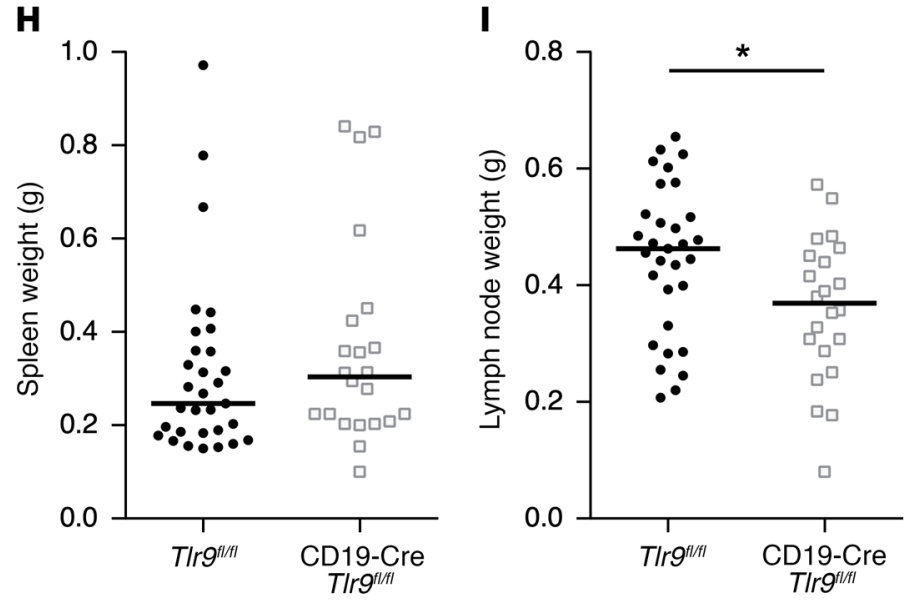
Figure 2. B cell-specific TLR9 deficiency results in exacerbated renal disease. (A) Schematic representation of T/rg floxed allele generation. Exons (open rectangles), LoxP sites (black triangles), FLP recombinase target sites (block open arrowheads) are also shown. (B) Sorted B cells from

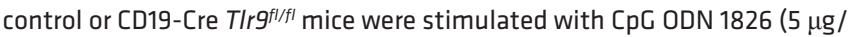
$\mathrm{mL}$ ) for 3 days and IgM secretion was quantified by ELISA ( $n=2$ per group). Scatter plots display data from individual mice with black lines showing means. ${ }^{* *} P<0.01$ using the 2-tailed Student's $t$ test. Phenotypic markers were assessed in 16-week-old MRL//pr mice from each indicated genotype, including (C) proteinuria, (D) glomerular renal disease, (E) interstitial and perivascular renal infiltrates, with $(\mathbf{F})$ representative images of H\&E kidney sections from mice of indicated genotype, where black arrowheads indicate interstitial inflammation and white arrows show glomeruli. Additional phenotypic endpoints were assessed for each noted genotype including (C) dermatitis, (H) spleen weight, and (I) lymph node weight. Controls: $n=24-31 ;$ CD19-Cre T/rg $g^{f / f l}: n=19-24$. Scatter plots display data from individual mice with black lines showing median values. ${ }^{*} P<0.05$, 2-tailed Mann-Whitney $U$ test.

from those of either preautoimmune (5-week-old MRL/lpr) or nonautoimmune mice $(\mathrm{BALB} / \mathrm{c})$. In all groups, the highest expression was observed in both DC lineages ( $\mathrm{DDC}$ and $\mathrm{pDCs}$ ), in which expression was 2.2- to 3.3-fold higher than in B cells. Within the B cell compartment, expression of TLR9 was similar in both marginal zone and follicular B cells (Figure 1). However, plasmablasts did not express TLR9 when compared with Tlr9-/ controls (not shown). Lack of Tlr9 mRNA expression in T cells was confirmed at the protein level, and despite reports to the contrary $(30,31)$, neutrophils did not exhibit TLR9 expression (Figure 1). BALB/c mice had statistically higher TLR9 expression in the CDC and macrophage compartments compared with diseased MRL/lpr mice. However, the overall pattern of TLR9 expression when comparing cell types was the same among different mouse strains (Figure 1). Furthermore, preautoimmune MRL/lpr mice exhibited no significant differences in TLR9 expression compared with older diseased MRL/lpr counterparts (Figure 1).

Generation of a Tlr9 conditional allele. Based on prior studies highlighting B cell-specific MyD88 as a major driver of SLE kidney disease (32), we postulated that $\operatorname{Tl} 9$ exerts its protective effects through its expression in B cells. Thus, to evaluate the role of $\operatorname{Tl} r 9$ in B cells, we generated a Tlr9-floxed allele and backcrossed it to the MRL/lpr lupus-prone background (Figure $2 \mathrm{~A})$. We then generated homozygous MRL/lpr Tlr $9^{A / / l}$ mice that were also heterozygous for CD19-Cre. Cre-negative $\operatorname{Tlr}^{f / / f l}$ littermates served as a negative control in this cohort. Tlr9 deletion in CD19-Cre $\operatorname{Tl} \operatorname{lr}^{f / / l}$ mice was assessed by qPCR of genomic DNA from FACS-sorted immune cell populations in 16-week-old mice. Tlr9 was efficiently deleted in B cells (96.3\% allele deletion) (Supplemental Table 1; supplemental material available online with this article; https://doi.org/10.1172/JCI132328DS1). As expected, TLR9 deletion was more than $10 \%$ in all other immune cell populations (Supplemental Table 1). Consistent with effective Tlr9 DNA deletion, CD19-Cre Tlr $9^{f / f l} \mathrm{~B}$ cells stimulated with CpG DNA demonstrated significant reduction in IgM secretion compared with controls (Figure 2B).

$B$ cell-specific deletion of TLR9 results in acceleration of murine SLE. To evaluate the impact of cell lineage-specific Tlr 9 deletion on disease state, several pathologic indicators of disease were assessed in 16-week-old mice. Similar to MRL/ lpr mice with global Tlr9 deficiency $(7,8)$, the CD19-Cre $\operatorname{Tlr} 9^{f / f l}$ mice exhibited significant increases in proteinuria and exacerbated glomerular and interstitial nephritis compared with Cre- littermate controls (Figure 2, C-F). No differences in dermatitis were identified across genotypes (Figure $2 \mathrm{G}$ ). Global Tlr9-deficient MRL/lpr mice exhibit pronounced splenomegaly and lymphadenopathy, while CD19-Cre Tlr $9^{f / f l}$ mice exhibited modest but statistically significantly decreased lymph node weight compared with littermate controls and demonstrated no differences in spleen weight (Figure 2, H and I). This suggests that the observed exacerbated renal disease is regulated at least in part independently of lymphoproliferation. To control for a possible effect of haploinsufficiency of CD19 (as the CD19-Cre is a knockin allele resulting in a deletion of 1 copy of CD19), CD19-Cre MRL/lpr mice were compared with MRL/ $l p r$ WT mice for disease outcomes. CD19-Cre MRL/lpr mice did not exhibit any significant differences in renal disease, dermatitis, lymph node weight, or spleen weight (Supplemental Figure 1A).

B cell-specific TLR9 deletion alters the autoantibody response. Serum anti-nuclear antibody (ANA) profiles were significantly altered by B cell-specific Tlr9 deletion with complete loss of homogenous nuclear staining typical of anti-chromatin antibodies and increased cytoplasmic, nucleolar, and fine-speckled staining indicative of RNA-related specificities (Figure 3, A and B), mirroring the ANA alterations previously documented in globally Tlr9-deficient lupus-prone strains (7). Consistent with the loss of homogenous staining pattern, the anti-nucleosome antibody titers were significantly reduced in the CD19-Cre $\operatorname{Tlr}^{f / / f l}$ group compared with controls. Titers of RNA-associated autoantibodies, such as anti-Smith and anti-RNA, remained unchanged (Figure 3C). These autoantibody patterns were attributable to Tlr9 deletion, as they were not observed in mice carrying the CD19-Cre allele alone (Supplemental Figure 1B). Thus, TLR9 expression in $\mathrm{B}$ cells is required for the development of autoantibodies to DNAassociated autoantigens.

Effects of B cell-specific TLR9 deletion on splenic T cell activation markers. CD19-Cre Tlr $9^{f / f l}$ mice exhibited fewer $\mathrm{CD} 19^{+}$cells as a percentage of the total splenocytes (Supplemental Table 2), which is attributable to a concomitant increase in the frequency of double-negative T cells (not shown). Notably, Tlr9-intact control mice had increased percentages of $\mathrm{CD} 44^{\mathrm{lo}} \mathrm{CD} 62 \mathrm{~L}^{\text {hi }}$ naive $\mathrm{CD} 4$ and CD8 cells compared with CD19-Cre Tlr $9^{f / / l}$ mice, suggesting increased activation of the $\mathrm{T}$ cell population when $\mathrm{B}$ cells lack TLR9 (Supplemental Table 2). Since CD19-Cre Tlr (f/l/l $^{\pi}$ mice had larger spleens, there was no net change in absolute cell number of naive CD4 and CD8 cells (Supplemental Table 3). Mice carrying the CD19-Cre allele without the Tlr $9^{\nexists / / f}$ genotype were not significantly different from WT MRL/lpr mice in the T cell compartment or in any of the disease assessments (Supplemental Figure 1C).

Myeloid-specific TLR9 deficiency did not alter clinical parameters of SLE pathogenesis. Although the CD19-Cre Tlr $9^{f / f l}$ mice recapitulated the exacerbated nephritis observed in global Tlr9deficient MRL/lpr mice, it remained possible that Tlr9 expression in other cell lineages contributes to SLE pathogenesis. TLR9 expression has been documented in myeloid lineages including cDCs, pDCs, neutrophils, and macrophages (10). Therefore, we 
A

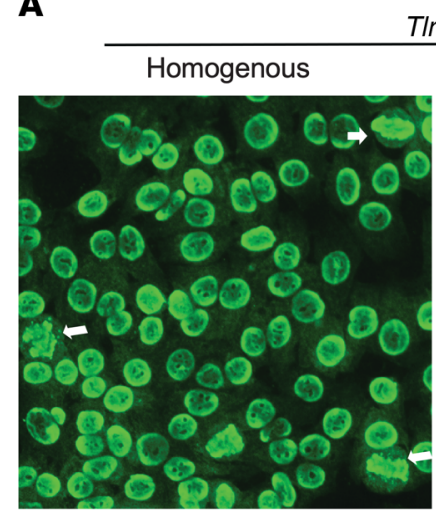

TIrgfl/fl
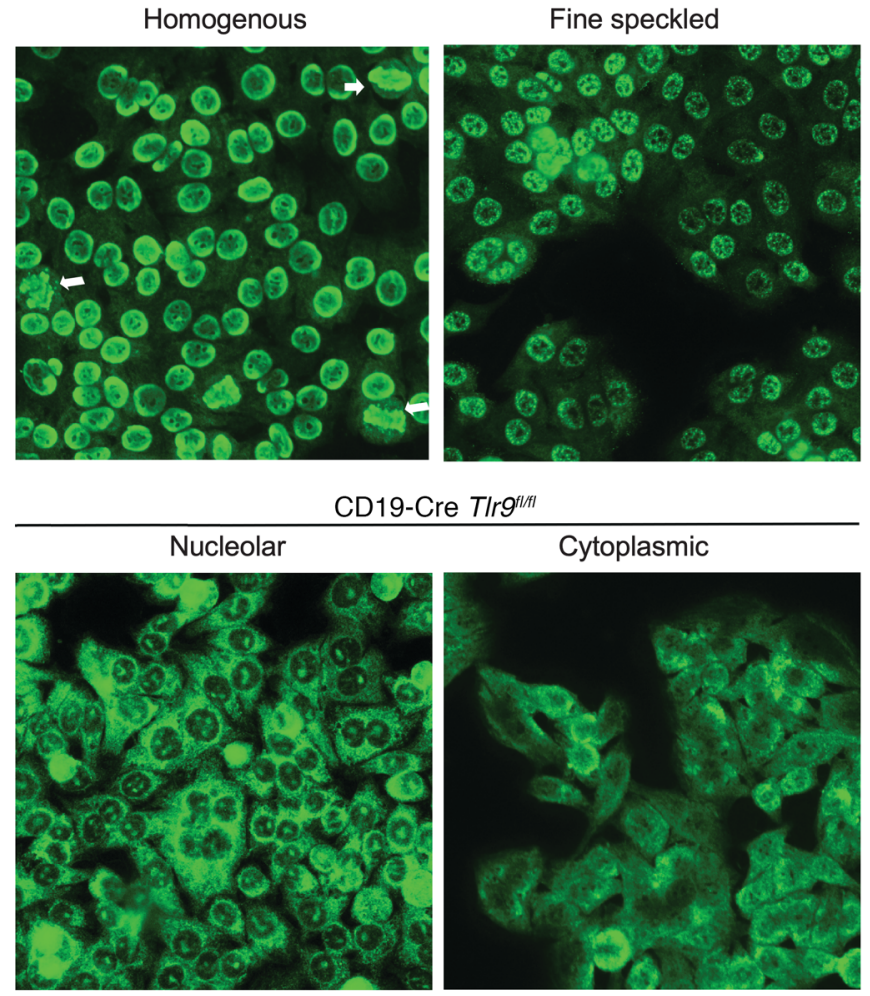

B

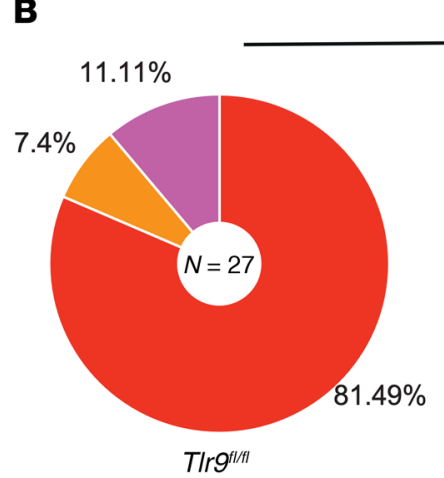

$\star \star \star \star$

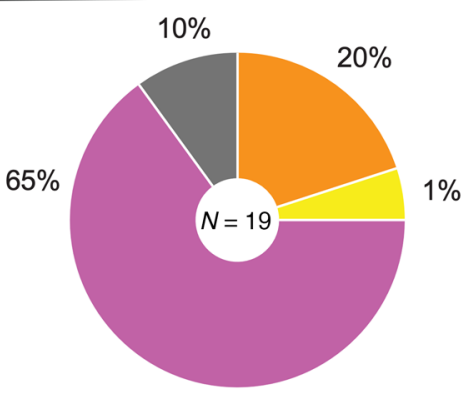

CD19-Cre TIrgt/tit
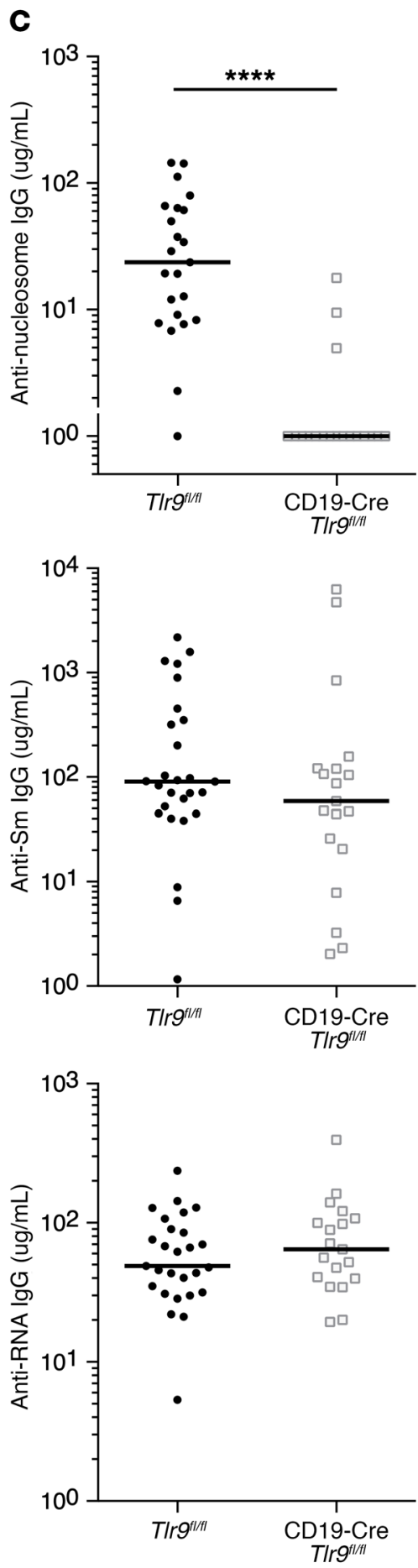

Figure 3. B cell-intrinsic deletion of TLR9 alters autoantibody patterns. (A) Representative HEp-2 ANA staining patterns from serum of control or CD19-Cre Tlrg ${ }^{f / f l}$ mice. Arrows indicate mitotic chromatin staining. Original magnification $\times 200$. (B) Frequency of ANA staining patterns produced by sera

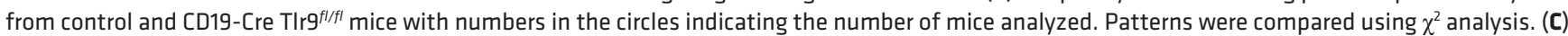
Serum concentrations of anti-nucleosome, anti-Sm, and anti-RNA IgC of control $(n=27)$ and CD19-cre TIr fl/fl $^{\prime}(n=19)$, as measured by ELISA. Scatter plots display data from individual mice with black lines showing median values. ${ }^{* * *} P<0.0001,2$-tailed Mann-Whitney $U$ test.

examined 3 additional promoter-driven Cre lines with myeloid tropism to assess the effect of TLR9 in myeloid lineages on clinical parameters and immune activation, including autoantibody production, in SLE. We used CD11c-Cre to delete TLR9 in cDCs and $\mathrm{pDCs}$, and MRP8-Cre to target neutrophils. To date, there is no Cre line that specifically and efficiently targets all macrophage subpopulations. Therefore, we employed LysM-Cre, which is predominantly expressed in macrophages and neutrophils, and MRP8-Cre, which is predominantly expressed in neutrophils, to deduce the differential contribution of macrophage and neutro- 
A
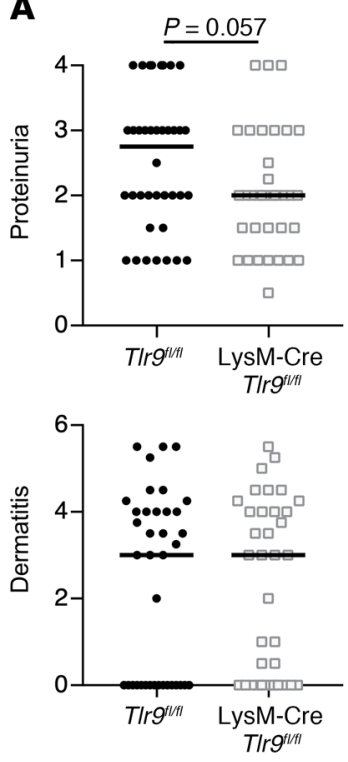

B
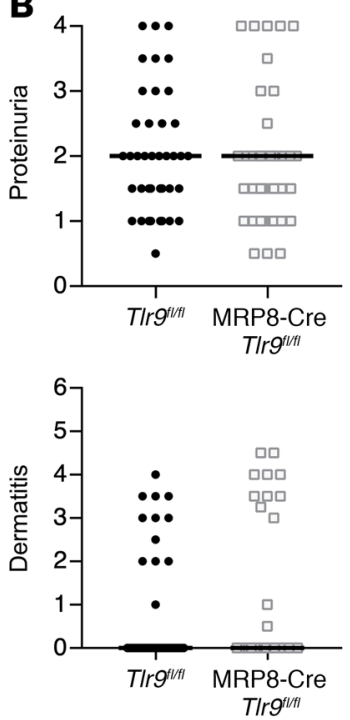

C
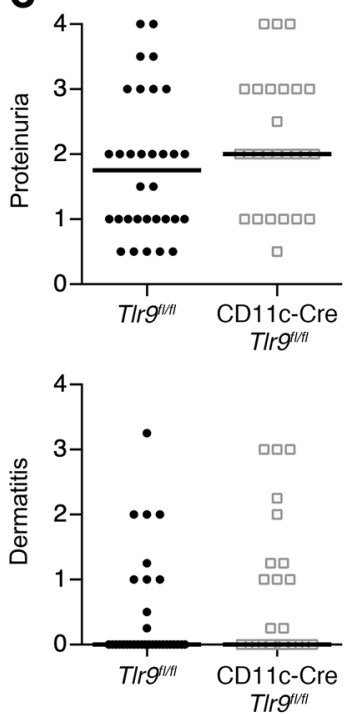
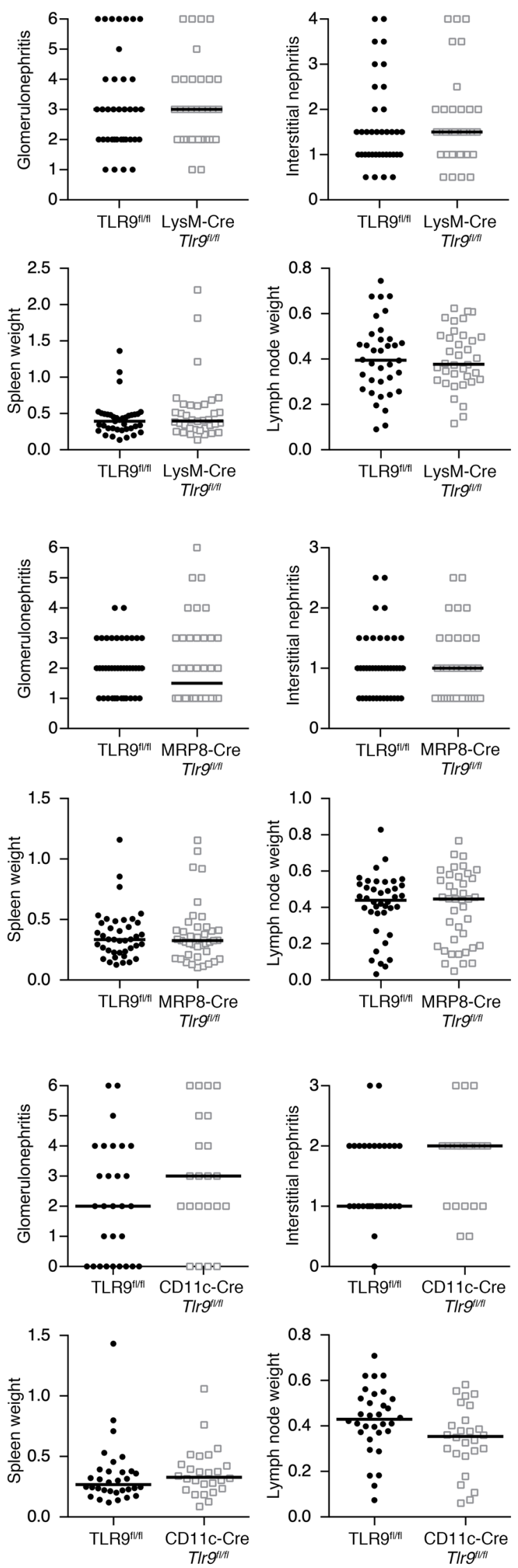

Figure 4. Myeloid-specific TLR9 deficiency does not alter clinical parameters of SLE pathogenesis. Evaluation of phenotypic markers of disease including proteinuria, glomerulonephritis, interstitial and perivascular renal infiltrates, dermatitis, spleen weight, and lymph node weight for the indicated Cre lineages.

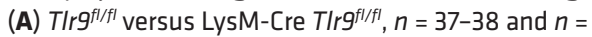
36 per group, respectively. (B) $T / r g^{f / f l}$ versus MRP8-Cre $T / r g^{f l / f l}, n=40$ per group, and (C) T/r $g^{f / f l}$ versus CD11c-

Cre TIr $g^{f l / f l}, n=26$ and $n=32$ per group, respectively, for phenotypic markers and $n=22$ and $n=29$ for histologic scoring. Scatter plots display data from individual mice, with black lines representing median values. No comparisons were statistically significant by 2 -tailed Mann-Whitney $U$ test.

phil TLR9 in lupus. For each cohort, mice were homozygous for the $\operatorname{Tlr} 9^{f l}$ allele and either heterozygous or negative for the indicated Cre.

LysM-Cre was previously reported to target a reporter gene in $80 \%$ of polymorphonuclear cells, $40 \%-50 \%$ of inflammatory monocytes, and $30 \%-40 \%$ of splenic macrophages (33). To assess the deletion of Tlr9 in our MRL/lpr system, we used quantitative PCR (qPCR) of genomic DNA prepared from FACS-sorted splenocytes. In LysM-Cre Tlr $9^{f f l}$ mice, deletion of TLR9 evaluated by qPCR on genomic DNA was $58.2 \%$ in macrophages $\left(\mathrm{CD} 11 \mathrm{~b}^{+} \mathrm{F} 4 / 80^{+}\right)$, 69.3\% in neutrophils (CD11b $\left.{ }^{+} \mathrm{Ly} 6 \mathrm{G} / \mathrm{C}^{+}\right), 26.5 \%$ in cDCs, and less than $15 \%$ in other assessed lymphoid lineages (Supplemental Table 1). Notably, these efficiencies may underestimate deletion in tissue-resident macrophages, since targeting rates of $90 \%$ or more were reported for LysM-Cre in peritoneal and alveolar macrophages (33).

We assessed clinical parameters of SLE in LysM-Cre Tlr $9^{f / / f l}$ mice, including renal disease, dermatitis, and lymphoproliferation. No differences in dermatitis were identified across genotypes (Figure 4A). A trend toward reduced proteinuria $(P=0.057)$ was observed in LysMCre $\operatorname{Tl} 9^{f / f l}$ mice when compared with $\mathrm{Cre}^{-}$littermate controls. This trend toward improved proteinuria in the setting of Tlr9 deficiency is opposite of what is seen in global Tlr9 deficiency. Moreover, we did not identify differences in either glomerulonephritis or interstitial disease (Figure 4A). Lymph node and spleen weights were similar among all genotypes (Figure 4A). No changes in autoantibody formation were observed in these mice (Figure 5A), nor did Tlr9 deletion by LysM-Cre alter the composition of the myeloid or lymphoid compartments in $\mathrm{MRL} / \mathrm{lpr}$ mice (Supplemental Table 2).

Although we observed no basal expression of TLR9 in neutrophils in MRL/lpr mice (Figure 
A
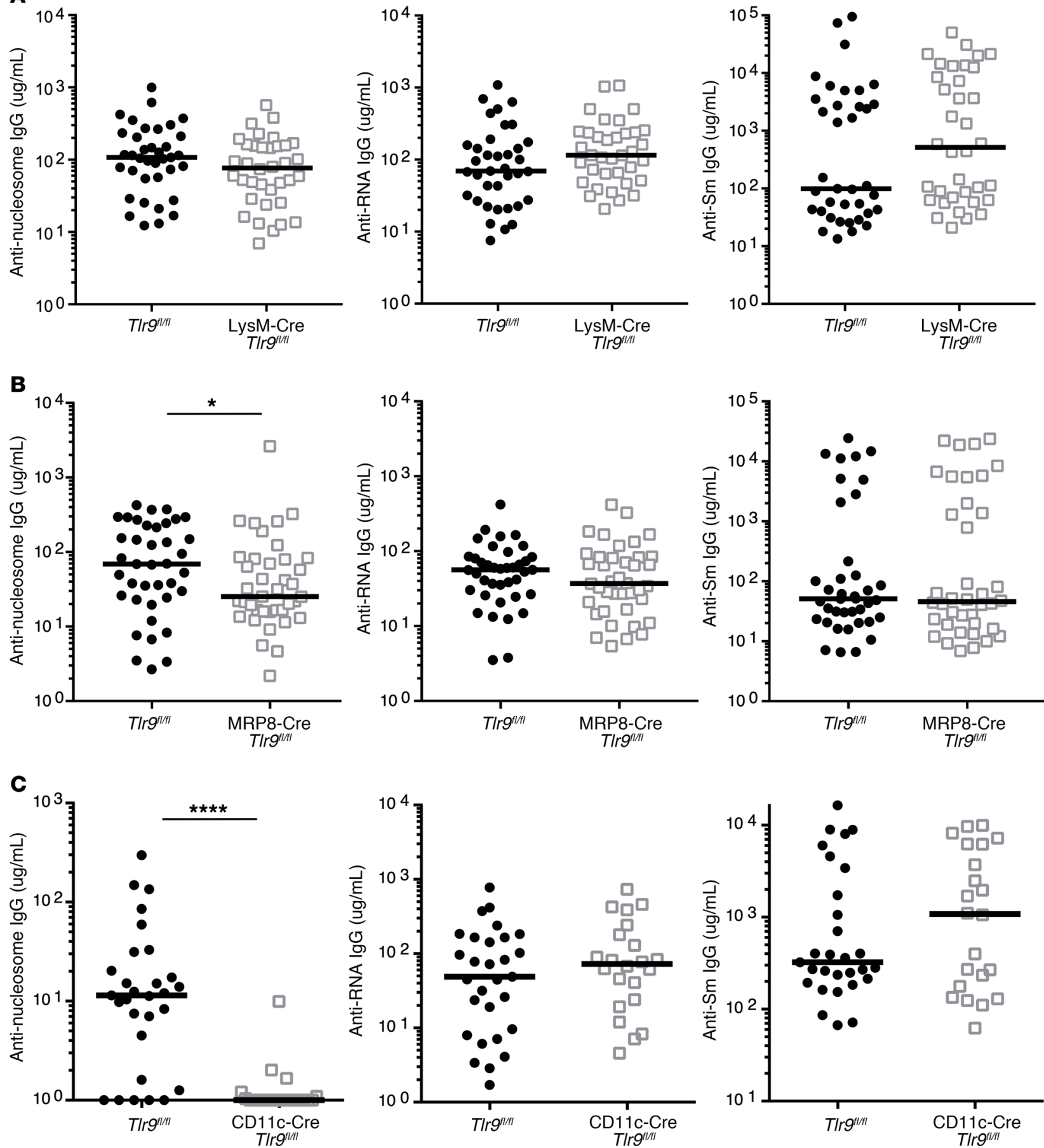

D

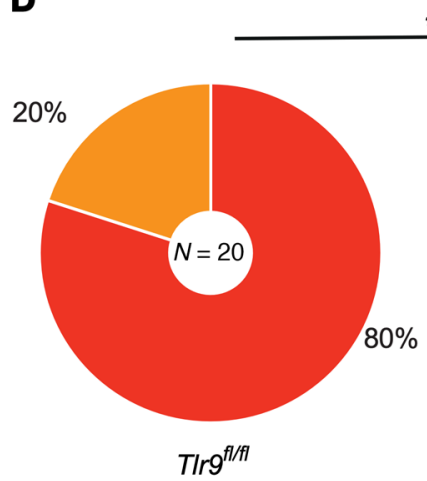

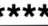

$11.76 \%$

Homogenous

Fine speckled

Cytoplasmic

Nucleolar

Other 
Figure 5. Alterations in autoantibody production after T/r9 deletion in myeloid cell lineages. Serum concentrations of anti-nucleosome, antiSm, and anti-RNA IgG from (A) T/r $9^{f / / f l}$ versus LysM-Cre T/r $9^{f / / f l}, n=37$ and $n=36$ per group, (B) T/rgf/ffl versus MRP8-Cre T/r $g^{f / / f l}, n=40$ and $n=39$, respectively, per group, and (C) T/r $g^{f / / f l}$ versus CD11c-Cre $T / r^{f / / f l}, n=22$ and $n$ $=29$ per group respectively. Scatter plots display data from individual mice with black lines representing median values. ${ }^{*} P<0.05$; ${ }^{* * * *} P<0.0001$, 2-tailed Mann-Whitney $U$ test. (D) Frequency of ANA staining patterns

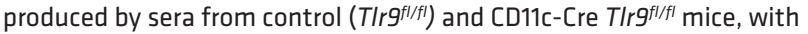
numbers in the circles indicating the number of mice analyzed. ${ }^{*} P<0.05$; ${ }^{* * * *} P<0.0001$, using $\chi^{2}$ analysis.

1, F and G), we felt it prudent to evaluate the role of neutrophil Tlr9 in lupus, given numerous reports suggesting Tlr 9 is expressed and functional in neutrophils $(22,34,35)$. Using a neutrophil-specific Cre (MRP8-Cre), Tlr9 deletion efficiency was $90.6 \%$ in neutrophils, with only minor effects on other cell lineages (Supplemental Table 1). No alteration of pathophysiology in the MRP8-Cre Tlr $9^{A / / 1}$ mice was noted when compared with littermate controls for any of the assessed parameters, including renal disease, dermatitis, and lymphadenopathy or splenomegaly (Figure 4B). MRP8-Cre $T \operatorname{lr} 9^{f / f}$ mice did exhibit mildly reduced anti-nucleosome titers. However, no differences were observed for anti-Sm and anti-RNA autoantibodies (Figure 5B). Tlr9 deletion in neutrophils did not substantially alter the composition of the myeloid or lymphoid compartments. Minor but significant alterations were observed in the $\mathrm{CD} 4^{+} \mathrm{T}$ cell compartment with an increase in the percentage of $\mathrm{CD} 4^{+} \mathrm{T}$ cells, as well as naive $\mathrm{CD} 4^{+} \mathrm{T}$ cells within the $\mathrm{CD} 4^{+} \mathrm{T}$ cell compartment (Supplemental Table 2). None of these minor changes would be explanatory of the global Tlr9 deletion phenotype of exacerbated disease.

pDCs and cDCs express high amounts of TLR9 protein and mRNA compared with other cell types (Figure 1). pDCs have been implicated in SLE pathogenesis as major producers of type 1 IFNs following stimulation by TLR7 or TLR9. To assess the role of these lineages in TLR9-mediated SLE pathogenesis, we crossed the $\operatorname{Tlr}^{f}{ }^{f}$ allele to the CD11c-Cre allele on the MRL/lpr background. The CD11c-Cre depleted greater than $90 \%$ of Tlr9 alleles as assessed by qPCR in sorted cDCs and pDCs. As previously reported, this Cre line can be promiscuous and also targets B cells $(33,36)$. In accordance with these data, we observed $93.2 \%$ deletion efficiency in plasmablasts, and $62.8 \%$ allele deletion in the B cell compartment. Despite the extensive deletion of Tlr9 in pDCs, cDCs, and plasmablasts, the CD11c-Cre Tlr $9^{\nexists / /}$ mice did not exhibit significant alterations in any of the measured clinical parameters of SLE, including proteinuria, glomerulonephritis, interstitial inflammation, dermatitis, or lymphoproliferation (Figure 4C). CD11c-Cre $\operatorname{Tl}^{\prime} \mathrm{f}^{\mathrm{A} / \mathrm{l}}$ mice had a substantial reduction in anti-nucleosome antibodies (a subtype of anti-DNA antibody) and an altered ANA pattern, comparable to the antibody pattern observed in CD19-Cre Tlr9-depleted mice (Figure 5, C and D, and Figure 3). This is consistent with extensive deletion of Tlr9 mediated by CD11c-Cre in plasmablasts. There was also a significant increase in plasmablasts in CD11c-Cre $\mathrm{Tlr}^{f / / f}$ mice, despite high deletion levels in this compartment, consistent with prior data (ref. 37 and Supplemental Tables 1 and 2).

$B$ cell-specific overexpression of TLR9 ameliorates disease in murine SLE. Given that suppression of TLR9 signaling via genetic deletion resulted in exacerbated disease, we hypothesized that TLR9 overexpression would result in ameliorated disease. To test this hypothesis, we created what we believe to be a novel murine model in which a Tlr 9 conditional allele was targeted to the rosa26 locus (Figure 6A). The targeted allele has a floxed region comprising EGFP, a neomycin resistance cassette, and a transcriptional stop sequence, which is followed by HA-tagged TLR9. Upon Cre-mediated recombination, the floxed region is excised, resulting in loss of eGFP and Tlr 9 overexpression driven by the rosa26 promoter. The absence of eGFP denotes successful recombination and serves as a surrogate for TLR9 expression.

As B cell-specific loss of Tlr9 resulted in exacerbated disease, we chose to overexpress $T \operatorname{lr} 9$ in the B cell lineage using the CD19-Cre allele. The TLR9 overexpresser strain is referred to as CD19-Cre Rosa ${ }^{\text {Thr }}$. Tlr 9 gene expression was 2.0 \pm 0.15 -fold higher in FACS-sorted B cells from CD19-Cre Rosa $a^{\text {Trr }}$ mice compared with control mice, as measured by qPCR (Figure 6B), and the HA-tagged TLR9 was found in both the cleaved and uncleaved forms (Figure 6C). To test the function of the Rosa ${ }^{\text {Trt }}$ conditional allele, CD19-Cre Rosa ${ }^{\text {Trr }}$ B cells were stimulated with varying doses of TLR9 agonist CpG ODN 1826, which resulted in a 1.7- to 2.5 -fold increase in IgM secretion (Figure 6D). Using EGFP as a surrogate for TLR9 expression, the percentage of B cells escaping the complete TLR9 overexpression phenotype was found to be $16 \% \pm 9 \%$ (Figure $6 \mathrm{E}$ ). This is of particular importance, as B cell escape has been responsible for altered or loss of phenotype in several other genetic deficiency models of SLE. Previously, selective pressure in the $\mathrm{CD} 19-\mathrm{Cre} M y d 88^{f / / / l}$ mice and CD19-Cre $\mathrm{MHCII}^{\mathrm{A} / \mathrm{fl}}$ mice resulted in only $50 \%$ and $29 \%$ of AFCs, respectively, having deletion of target genes, compared with $90 \%$ of naive B cells $(32,38)$. This suggests that autoreactive B cells without protective mutations undergo increased expansion and differentiation. Despite this technical caveat, we aimed to determine whether even incomplete overexpression of TLR9 in B cells would result in altered disease.

We generated homozygous MRL/lpr Rosa ${ }^{\text {Tlr }}$ mice that were also heterozygous for CD19-Cre. In these experiments, CD19Cre-negative littermates served as controls. Indeed, as hypothesized, B cell-specific Tlr9 overexpression ameliorated disease in MRL/lpr mice. CD19-Cre Rosa ${ }^{\text {Trr }}$ mice exhibited a significant reduction in proteinuria and glomerulonephritis but no difference in interstitial disease (Figure 7, A and C-E). There was no difference between the groups in dermatitis, splenomegaly, or lymphadenopathy (Figure 7, B, F, and G). The composition of both the lymphoid and myeloid compartments was similar among all the groups (Supplemental Table 2). CD19-Cre Rosa $a^{\text {Tr }}$. mice demonstrated no change in serum anti-nucleosome autoantibodies (Figure 7H); nonetheless, they exhibited a modest but significant decrease in anti-RNA antibodies (Figure 7I). Notably, the ratio of anti-nucleosome/RNA antibodies within each animal was increased in B cell-specific Tlr9-overexpressing mice (Figure 7J), suggesting that the balance between TLR7 and 9 signaling had been altered.

To further confirm our findings in the MRL/lpr background and to determine whether overexpression of TLR9 could protect against a TLR7-driven disease, we crossed the Rosa $a^{\text {Trr }}$ allele to the B6.Fcgr2b $b^{-1}$.Yaa SLE model. In this model, the Yaa modifier 
A

pROSA26 TLR9
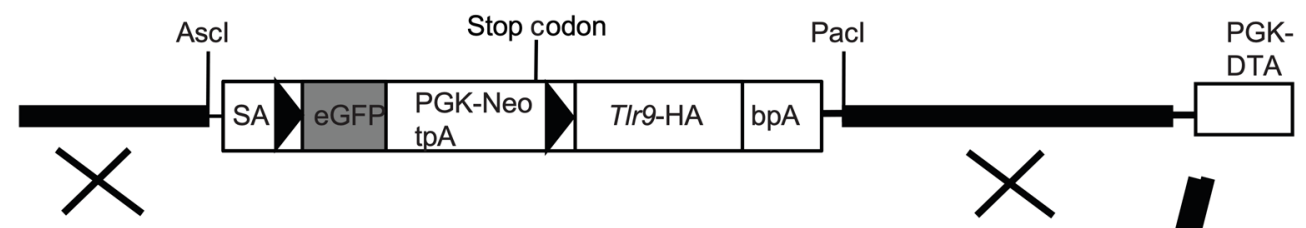

ROSA26 locus

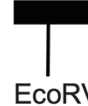

ROSA26 eGFP

TLR9 allele

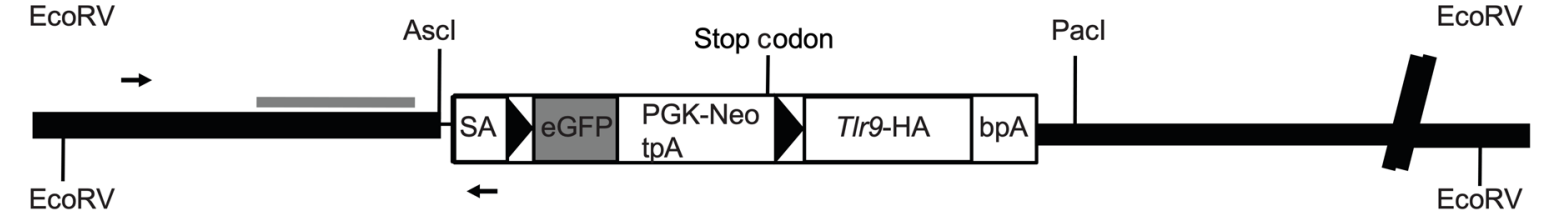

ROSA26 eGFP TLR9 allele after Cre mediated excision

B

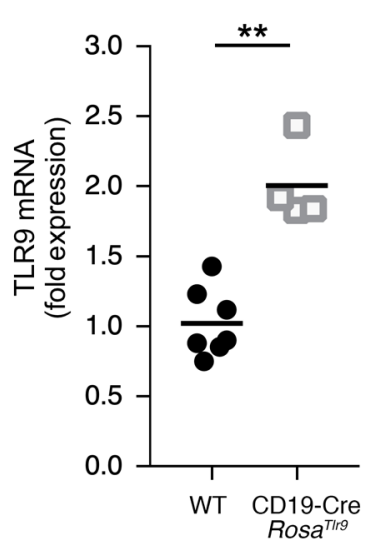

E

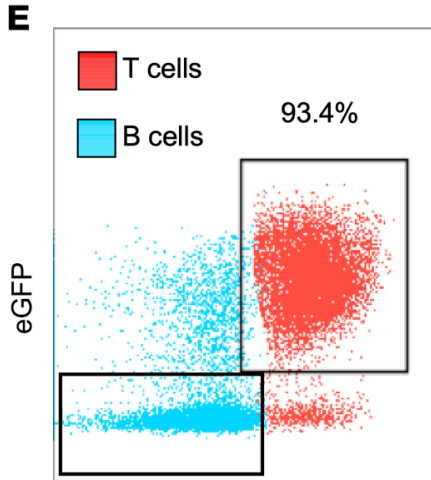

$80.1 \%$
C

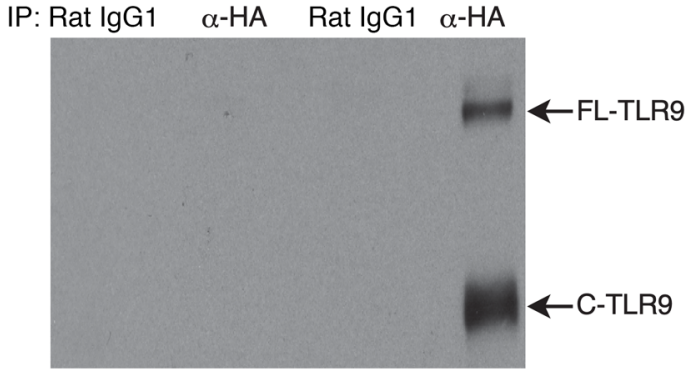

Immunoblot $\alpha-\mathrm{HA}$

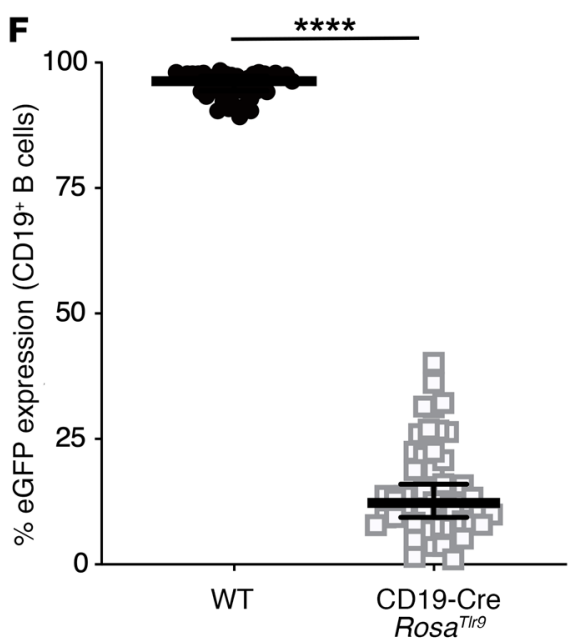

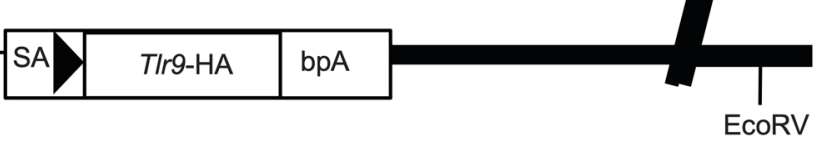

D $\square$ WT $\square \underset{\text { Rosat }}{\text { CD1/r9 }}$

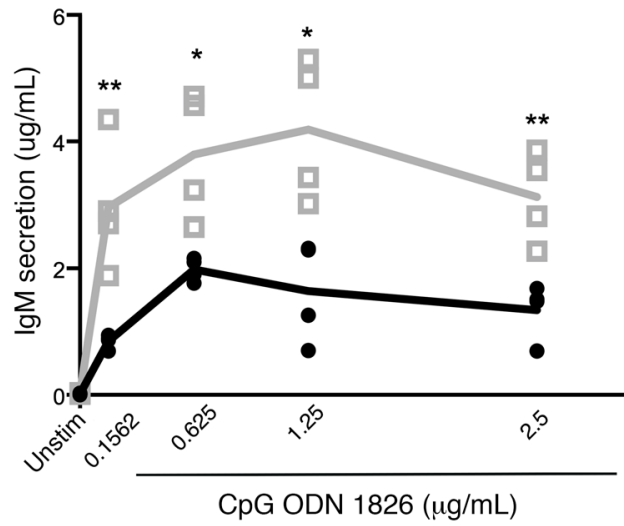


Figure 6. Generation and validation of a conditional TLR9 overexpression allele. (A) Diagram of the TLR9 overexpression plasmid and insertion into the rosa26 locus. The plasmid contains 2 rosa26 homology arms flanking the expression vector. The vector is composed of a floxed region (demarcated by black triangles) containing eGFP, a Neo cassette, and a transcriptional stop sequence. This floxed sequence is followed by an HA-tagged TIr9. PGK-DTA was used as a negative selection marker for ES cells; bPA represents the bovine growth hormone polyadenylation site. The top panels show the targeting plasmid and rosa26 locus, and the bottom 2 panels show the Rosa ${ }^{T r}$ locus before and after Cre-mediated excision. (B) qPCR analysis of TLR9 expression in sorted B cells from control $(n=7)$ and CD19-Cre Rosa ${ }^{\text {Trg }}$ mice $(n=4)$ mice. (C) Representative Western blot showing TLR9-HA expression in CD19-Cre Rosa ${ }^{\text {Trg }}$ mice but not control mice. Sorted B cells were immunoprecipitated (IP) with isotype control antibody (rat IgG1) or anti-HA antibody and immunoblotted with anti-HA antibody. Arrows depict the full-length (FL-TLR9) and cleaved (C-TLR9) forms of TLR9. (D) Sorted B cells from control and CD19-Cre Rosa ${ }^{T r 9}$ mice were stimulated with CpG ODN 1826 (at indicated concentrations) for 3 days and IgM secretion was measured by ELISA. Scatter plots display data from individual mice, with black lines showing means. (E) Left shows representative FACS plots showing GFP expression in CD19+ (red) and TCR $\beta^{+}$ cells derived from CD19-Cre Rosa ${ }^{\text {Trr }}$ mice, with the right panel showing summary data from CD19-Cre ${ }^{+}$and Cre-negative Rosa ${ }^{\text {TrP }}$ mice ( $n=42$ and $n=46$, respectively). For tabulated data, each dot denotes an individual mouse and horizontal lines represent the mean and standard deviation. ${ }^{*} P$ $<0.05$; ${ }^{* *} P<0.01$; ${ }^{* * *} P<0.0001$ using Student's $t$ test.

that harbors a duplication of TLR7 is responsible for amplifying the lupus pathology in the Fcgr $2 b^{-/-}$mouse strain (39). Similar endpoints were assessed in 24-week-old male mice, with the exception of dermatitis, which is not a feature of this genetic background. As with the MRL/lpr model, Fcgr $2 b^{-/-} . Y a a-R o s a^{\text {Tlr }}$ mice had a significant decrease in renal disease, as assessed by proteinuria and glomerulonephritis; in addition, they also demonstrated reduced interstitial nephritis (Figure 8, A-D). Spleen and lymph node weight remained unchanged, recapitulating the data from the MRL/lpr model (Figure 8, E and F, and Figure 7, F and G). There was no observed difference in autoantibody production, although all antibodies were observed at much lower titers than in the MRL/lpr model (Figure 7 and data not shown). Altogether, our results demonstrate that the severe glomerulonephritis manifested in Fcgr2b - $^{-1}$.Yaa mice due to a 2-fold increase in TLR7 was ameliorated by a 2-fold overexpression of TLR9 in B cells.

One potential mechanism for the regulation of TLR7 by TLR9 is that TLR9 may suppress TLR7 at the transcriptional level. To assess this, TLR7 mRNA was measured in the B cells of TLR9-deficient and -overexpressing mice. Although TLR9 mRNA was expressed at 2-fold-increased levels in the overexpressing mice, there was no effect on the expression of TLR7 (Supplemental Figure 2).

\section{Discussion}

The roles of TLRs and MyD88 have been well established in numerous models of SLE as well as in patients with SLE (1, 2, $40,41)$. A major unsolved puzzle with respect to TLR signals and disease pathogenesis is why and how TLR9 actually protects from disease, even as it promotes the hallmark lupus-associated anti-chromatin antibody response. To gain further insight into this paradox, we examined the cell type-specific effects of Tlr 9 deficiency. Despite the broad expression pattern of Tlr9 (and the use of multiple Cre lines that targeted lineages including B cells, DCs, neutrophils, and macrophages), only B cell-specific Tlr9 deficiency resulted in acceleration of lupus nephritis. Further, we found that in 2 independent models of SLE, Tlr9 overexpression in the B cell compartment resulted in ameliorated renal disease. Taken together, these results identify B cells as a primary and possibly unique site of TLR9-based regulation of multiple aspects of lupus-like autoimmunity.

Specific promoter-driven Cre lines commonly used to target immune cell lineages in fact affect multiple lineages and also often affect only a fraction of the intended target cells, making interpretation more complicated. Moreover, not all target floxed loci are equally affected by a given Cre line (42). Here we have been careful to directly measure the extent of deletion in relevant lineages using qPCR of genomic DNA to help clarify conclusions. In addition, our strategy of using multiple Cre lines that inevitably overlap is helpful when taking the data together for interpretation.

From these data we can conclude that TLR9 expression in both pDCs and cDCs is not likely to be important in regulating lupus in MRL.Fas ${ }^{l p r}$ mice. Deletion was extensive in both lineages using the CD11c-Cre, yet no significant clinical effects were observed. A potential complication of CD11c-Cre is substantial off-target effects in B lineage cells, in particular plasmablasts. But as our CD19-Cre mice did show a phenotype, while the CD11c-Cre did not, we can conclude that TLR9 expression in pDCs and CDC TLR9 does not contribute to disease regulation. Neutrophils were also extensively targeted by LysM-Cre, yet we saw no effect on disease. This was corroborated by the MRP8-Cre mice that targeted neutrophils with reasonable efficiency but much more specifically. From these 2 crosses we can conclude that neutrophil-expressed TLR9 is also unlikely to regulate lupus.

The role of Tlr9 in macrophages is harder to interpret, since only one Cre (LysM) affected them, and then only to a partial degree. This Cre line is reported to variably affect macrophages depending on their location, so it is conceivable that there was more extensive deletion in some tissue macrophages that we did not assay (33). Nonetheless, it remains possible that macrophage-expressed TLR9 could still regulate disease in addition to B cell-expressed TLR9. This will not be resolvable until the advent of Cre lines with both greater efficiency and specificity within the macrophage lineages. Additionally, our data do not rule out a role for Tlr9 in nonhematopoietic lineages. However, recent work showed that Tlr9 expression on renal tubular epithelial cells promotes acute kidney injury, albeit in a nonautoimmune model, suggesting parenchymal Tlr9 is likely not renal-protective (43).

The importance of TLR regulation in B cells, though supported by other data, was not completely anticipated. For example, TLR7 overexpression in cDCs was identified as an important driver of nephritis in 2 different B6-based models $(44,45)$. However, overexpression can show what is sufficient, but does not necessarily pinpoint what is happening at more physiological levels of expression. In our case, to the extent that TLR7 is constrained by TLR9, deletion of TLR9 in CDCs was not sufficient to drive more disease in a model where global TLR9 deletion was sufficient to drive disease in a TLR7-dependent manner (7). Nonetheless, others and we do identify a unique role for DCs per se in promoting nephritis (25, 


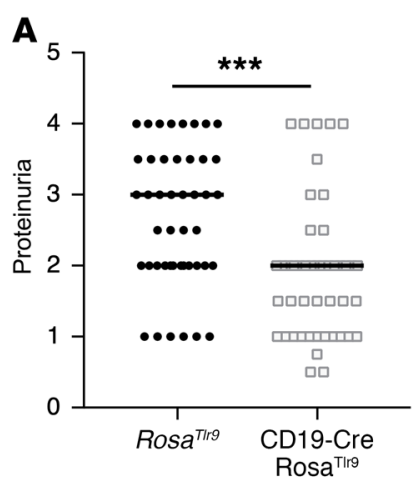

E

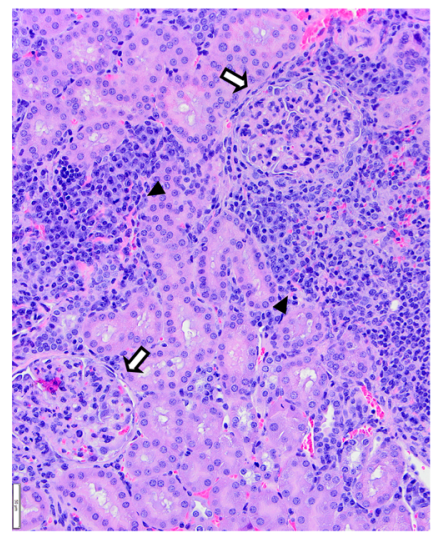

$\operatorname{Rosa}^{T r 9}$

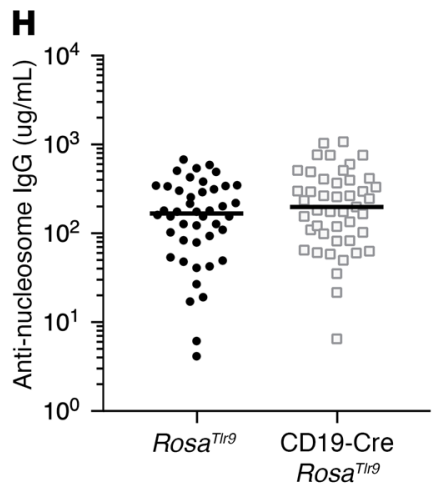

$\mathbf{B}$
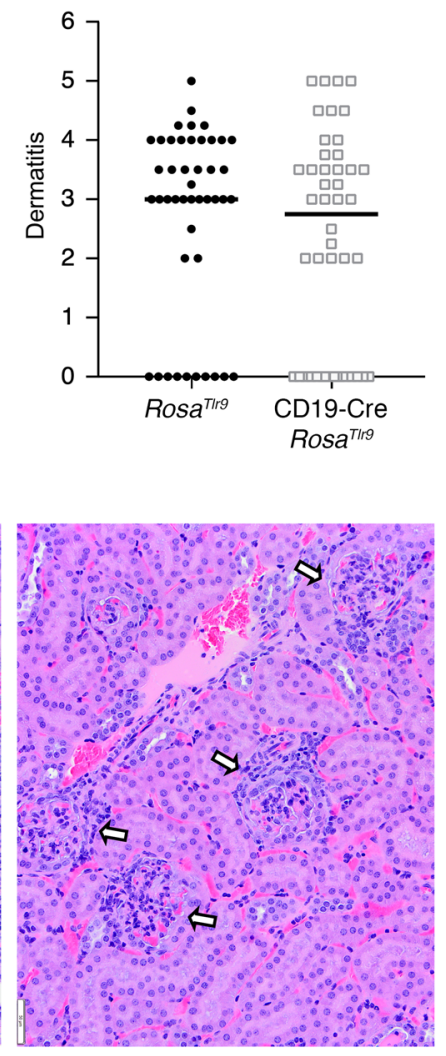

CD19-Cre Rosa ${ }^{\text {Tr9 }}$

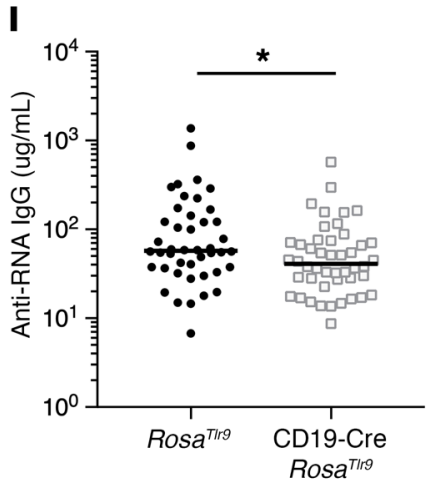

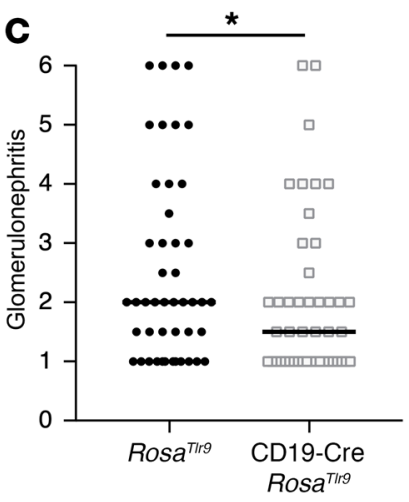
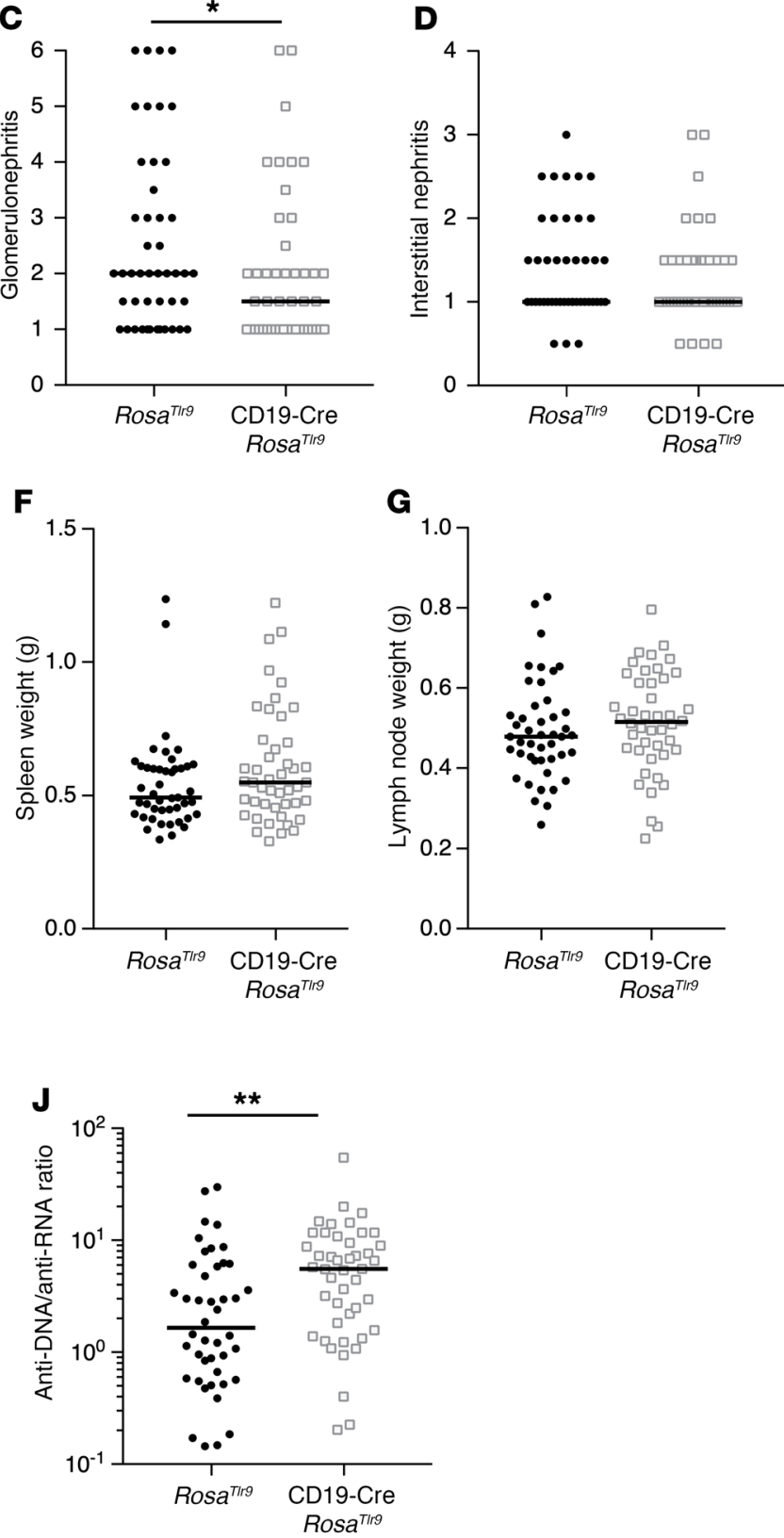

Figure 7. B cell-specific overexpression of TIr9 results in ameliorated renal disease and altered antibody profile in MRL/Ipr mice. CD19-Cre Rosa ${ }^{T / 9} \mathrm{MRL} / / \mathrm{pr}$ and Cre-negative Rosa ${ }^{T r}{ }^{T / 9} \mathrm{MRL} / \mathrm{lpr}$ controls were aged until 19 weeks (female) and 21 weeks (male). Phenotypic markers were assessed, including (A) proteinuria, (B) dermatitis, (C) glomerular renal disease, and (D) interstitial and perivascular renal infiltrates, with (E) representative images of H\&E kidney sections from mice of indicated genotypes, where black arrowheads denote interstitial inflammation and white arrows show glomeruli. Original magnification $\times 200$. Additionally, (F) spleen weight and (C) lymph node weight were assessed as markers of lymphoproliferation. Serum concentrations of (H) anti-nucleosome, (I) anti-RNA antibody formation, and (J) ratio of anti-nucleosome/anti-RNA antibodies of Rosa ${ }^{T / r 9}$ controls $(n=45)$ and CD19-Cre Rosa ${ }^{\text {Trg }}(n=46)$. Scatter plots display data from individual mice with black lines showing median values. ${ }^{*} P<0.05$; ${ }^{* *} P<0.01$; ${ }^{* *} P<0.001,1$-tailed Mann-Whitney $U$ test.

45). Previously, we deleted MyD88 in B cells and found markedly reduced disease. This indicated that proinflammatory TLR effects in B cells were important (32). Presumably, these were driven by TLR7 in the B cell, though that has yet to be directly tested. Our current results nicely complement these prior studies by showing that TLR9 is required in the B cell to restrain disease.

Previously, using a Wiskott Aldrich syndrome protein (WASp) model of autoimmunity, Jackson et al. showed that B cell-intrinsic loss of TLR9 resulted in exacerbated disease, consistent with our findings (13). However, we note that this model depends on the loss of a single gene that is not implicated in lupus (in contrast to our multigenic MRL model) and, most importantly, that this loss of WASp was itself restricted to B cells in this model. The use of a model driven solely by the loss of a single gene in B cells may render it as a foregone conclusion that TLR expression in B cells would also be important in this context. For these reasons, it would be hard to generalize from this model to more polygenic models in which multiple cell types play a role in pathogenesis. Nonetheless, 
agreement of disparate murine models tends to support the relevance of the basic mechanisms elucidated in the model systems.

Our studies, along with others, suggest that B cells that respond to TLR ligands control not just autoantibodies, but also nephritis and $\mathrm{T}$ cell activation. This conclusion comes from results of manipulating MyD88, TLR7, or TLR9 in B cells $(13,32)$, which should affect only nucleic acid-specific B cells, yet has broad global effects on disease. These considerations in turn imply there is a mechanism of tissue pathogenesis that stems from initial activation of nucleic acid-specific B cells.

At the molecular level, TLR9 likely regulates TLR7 in the same $\mathrm{B}$ cell, consistent with in vitro data suggesting disparate roles for TLR7 and TLR9 in B cell-specific activation, proliferation, death and differentiation upon stimulation of both BCR and TLR $(37,46)$. Specifically, studies of TLR signaling in the B cell compartment suggest that TLR9 stimulation limits expansion of autoreactive B cell populations (46) and is required for spontaneous peripheral activation of anti-DNA B cells, as well as their differentiation into Ab-producing cells $(37,47)$. Therefore, the observed Tlr9 protective effect we observe herein may be due, at least in part, to regulation of these autoreactive B cells. It has also been suggested that TLR9 competes with TLR7 for the chaperone Unc93b that is required for proper processing, transport, and expression of both TLRs, and that in the absence of TLR9, there is enhanced TLR7 activity (48-52). However, these studies have been conducted in cell lines or using global mutations in vivo. Most studies of TLR9 signaling per se have focused on macrophages, although studies in pDCs and one study in B cells suggests marked cell type specificity in the nature of the signaling pathways (53-55). These differences could underlie in part why we observed only a regulatory role in vivo in B cells. Our implication of TLR9 as a key regulator operating directly in B cells emphasized the importance of studying TLR9-TLR7 interactions in B cells, which have been scarcely investigated to date (53).

TLR9 could also be regulating TLR7-driven autoimmunity in trans, by promoting the production of protective anti-chromatin antibodies. This concept may seem to disagree with the consensus in the lupus field that anti-DNA and related autoantibodies are disease mediators $(56,57)$. However, the current study and our prior work suggest that this may not always be the case. First, B cell-specific Tlr9-deficient mice exhibit exacerbated disease, despite the fact that they do not produce any anti-nucleosome antibodies. Similar observations were made in the context of global Tlr9 deficiency in multiple murine mouse models of SLE (11-17). It is thought that the specific loss of anti-nucleosome antibodies likely is due to the fact that the BCR and TLR9 receptors signal in concert and loss of TLR9 prevents the second signal needed for the BCR ligation to result in autoantibody production (58). Additionally, Tlr9 overexpression ameliorated disease without a concomitant change in anti-nucleosome antibodies and only a slight decrease in anti-RNA antibodies. Of interest in this regard from the current work is the observation that both CD19cre and CD11c-Cre Tlr $9^{f / f l}$ mice exhibit a loss of dsDNA antibodies, but only CD19-Cre mice exhibit ameliorated pathology. This unlinking of the autoantibody response from end-organ damage is consistent with some prior results, for example that lupus-prone mice that cannot make circulating autoantibodies but do have $\mathrm{B}$ cells still develop lupus nephritis (59). Moreover, some human and murine studies suggest that IgM anti-dsDNA antibodies are protective (60-62). Hence it remains an intriguing possibility that some TLR9-driven antibodies actually have a dominant protective effect, perhaps by promoting autoantigen clearance, partly explaining the regulatory role of TLR9.

Although TLR9 overexpression had a significant protective effect in 2 models of disease, the extent of this protection may have been limited by some technical aspects of the model system used. Both TLR9 overexpression alleles were not expressed in an average of $15.6 \%$ of the B cells, so there were some B cells that lacked the suppressive effect; these cells could have dominantly promoted disease, for example by serving as APCs for autoreactive $\mathrm{T}$ cells. There is precedent for this effect from escapee B cells when CD19-Cre was used to conditionally delete MHCII in MRL/lpr mice (38). Second, expression of 2 copies of the Rosa allele resulted in only a 2 -fold increase in expression of TLR9; even higher expression of TLR9 may have had greater protective effects. It is theoretically possible that the CD19-Cre allele and consequent CD19 haploinsufficiency may confer some protective effects, potentially accounting for some of the ameliorated disease in the CD19-Cre Rosa ${ }^{\text {Tlr }}$ mice. However, there was no significant disease amelioration or effects on any measured immunologic parameter when the CD19-Cre allele was studied alone in the MRL/lpr background. These data suggest that the effects of CD19-Cre per se in ameliorating disease in the context of promoting TLR9 overexpression would be limited, and thus, based on the data we have available, we conclude that TLR9 overexpression has a protective role in models of SLE.

In summary, we have delineated the role of B cell-specific TLR9 in SLE pathogenesis. B cell-specific Tlr9 deficiency is a disease accelerator in SLE pathogenesis. Further, our study is unique in demonstrating that B cell-specific Tlr9 overexpression resulted in ameliorated renal disease. These conclusions relate to emerging human studies demonstrating that B cells from lupus patients are hyporesponsive to TLR9 stimulation $(63,64)$. Our studies in turn suggest that TLR9 hyporesponsiveness in the $\mathrm{B}$ cells of these patients may represent loss of a protective mechanism, which results in initiation and/or potentiation of autoimmunity. Our findings further suggest the counterintuitive notion that TLR9 agonism may suppress lupus. Systemically administered TLR9 agonists have been used in the clinic in the context of cancer treatment, and were generally well tolerated $(65,66)$. It may be feasible to devise a strategy to deliver such agonists just to B cells or even to DNA-specific B cells. Hence, understanding how discrete cell populations regulate lupus may allow for more targeted therapeutic design.

\section{Methods}

Flow cytometry. Flow cytometry was performed as previously described (67). Spleens were processed via mechanical dissociation and lysed using ammonium-chloride-potassium buffer (prepared in house). Cells were resuspended in phosphate buffered saline (PBS) with $3 \%$ calf serum and the FcR-blocking antibody 2.4G2. Ethidium monoazide (EMA) or Ghost 510 (Tonbo) was used for live-dead discrimination. Cells were fixed in 1\% paraformaldehyde or Cytofix/ Cytoperm (BD Biosciences) where appropriate. Flow cytometry was performed on a BD Biosciences LSRII and cell sorting on BD FACS 
A

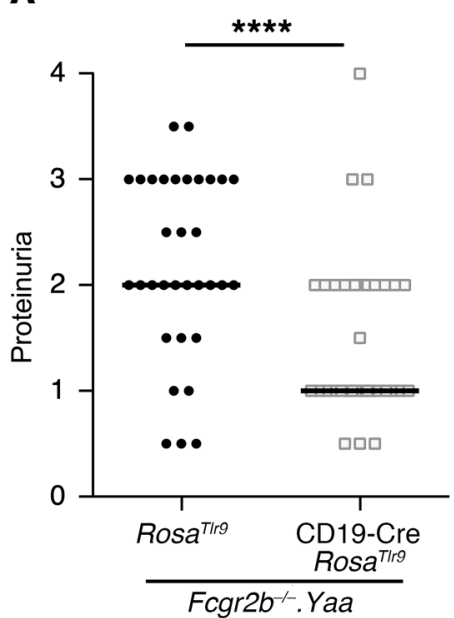

D
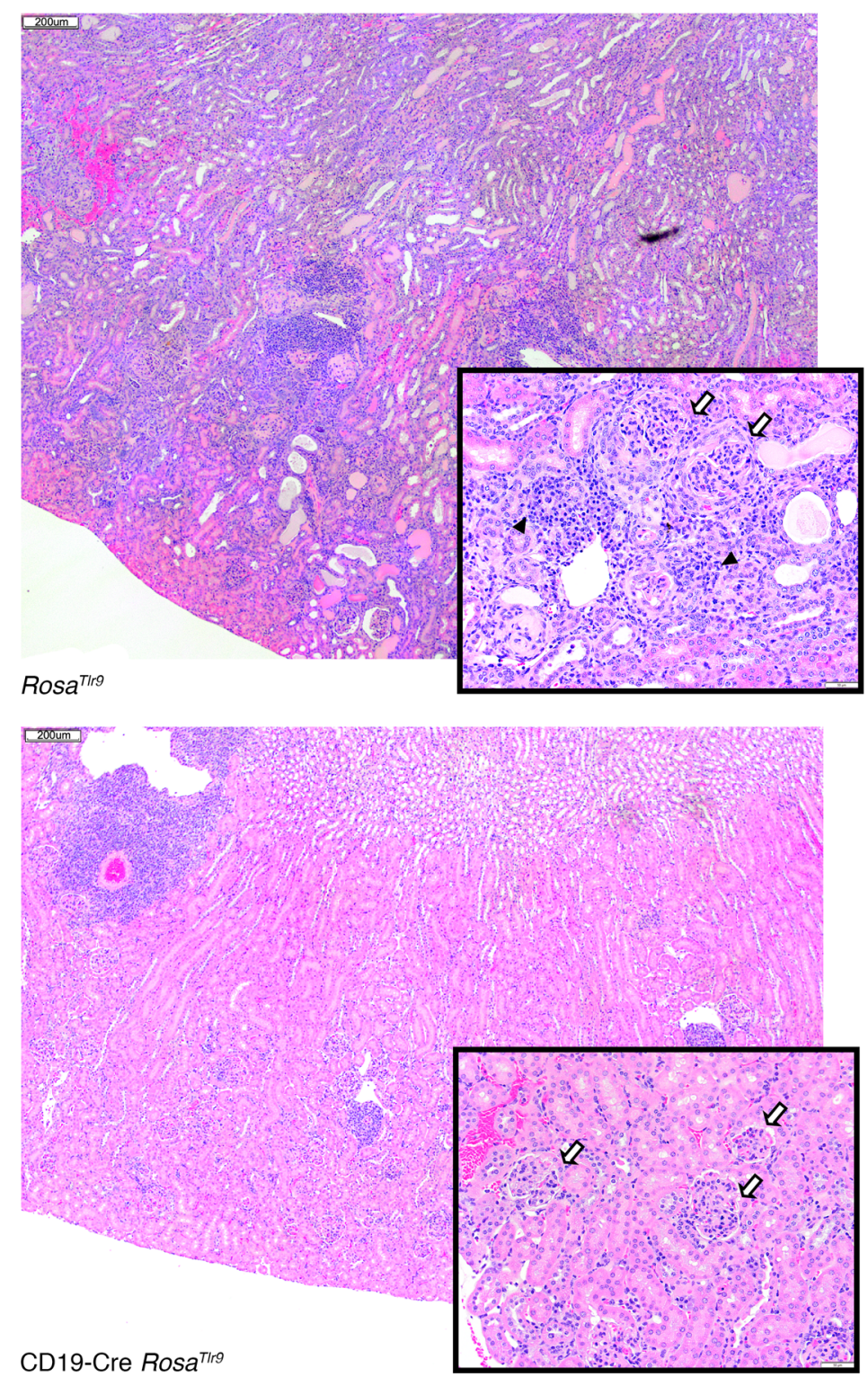

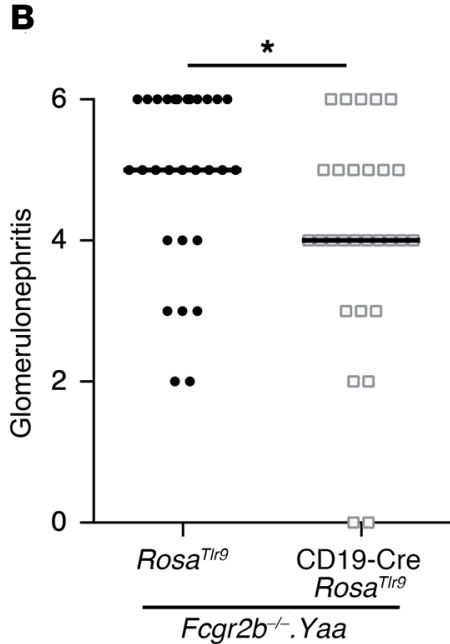

C

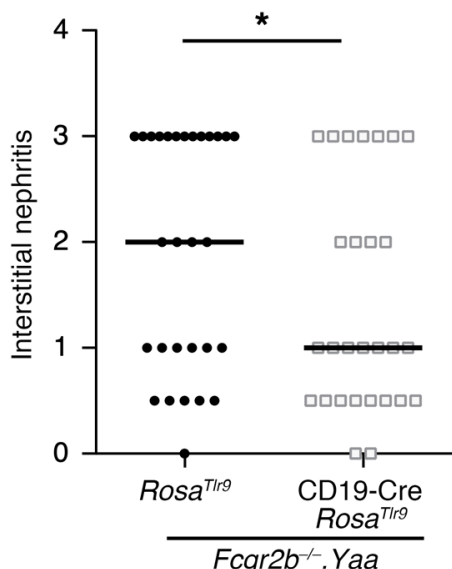

E

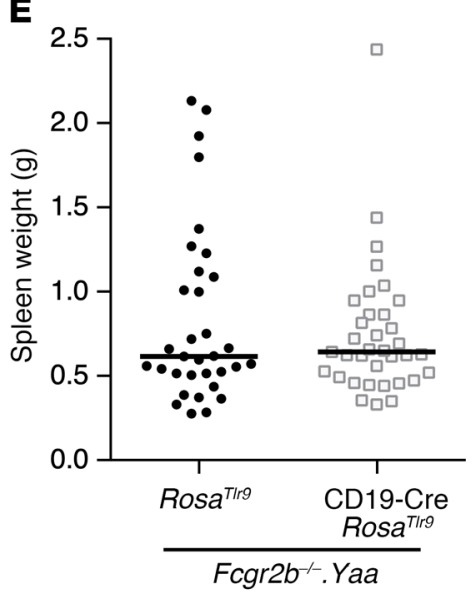

$\mathbf{F}$

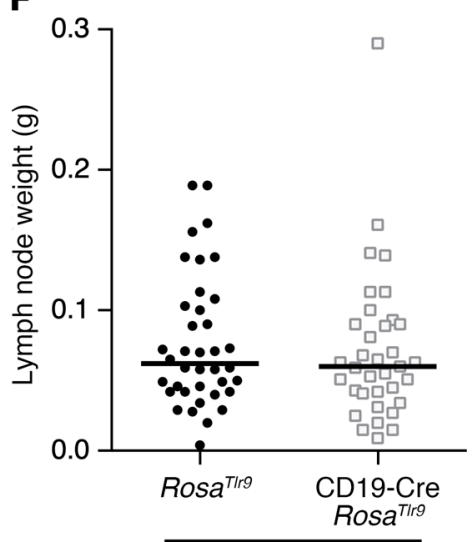

Fcgr2b ${ }^{-}$.Yaa 
Figure 8. B cell-specific overexpression of TIr9 results in ameliorated renal disease and altered antibody profile in $\mathrm{Fcgr2}^{-1 / .}, \mathrm{Yaa}$ mice. CD19-Cre Rosa $^{\text {Trl }} \mathrm{Fcgr2b}^{-/-}$.Yaa and Cre-negative Rosa ${ }^{\text {Trl }} \mathrm{Fcgr} \mathrm{b}^{-/ .}$.Yaa male controls were aged until 24 weeks. Phenotypic markers assessed included (A) proteinuria, (B) glomerular renal disease, and (C) interstitial and perivascular renal infiltrates, with (D) representative images of H\&E kidney sections from mice of indicated genotype, where black arrowheads indicate interstitial inflammation and white arrows show glomeruli. Original magnifications $\times 40$ and $\times 200$; scale bar: $200 \mu \mathrm{m}$. Additionally, (E) spleen weight and (F) lymph node weight were measured as markers of lymphoproliferation. Scatter plots display data from individual mice, with lines showing median values. ${ }^{*} P<0.05$; ${ }^{* * *} P<0.0001,2$-tailed Mann-Whitney $U$ test.

Aria II. Analysis was performed in FlowJo 10. Surface and intracellular staining antibodies are listed below.

Measurement of serum antibodies. HEp-2 immunofluorescence assays (Antibodies, Inc. or Bio-Rad) were performed as previously described (8) with serum diluted at 1:200. Images were captured on a Zeiss LSM 510 microscope and processed in Adobe Photoshop. Antinucleosome, anti-Sm, and anti-RNA concentrations were measured by ELISA as previously described (68). Specific antibodies were detected with alkaline phosphatase-conjugated goat anti-mouse IgG (Southern Biotech [1030-04]). The monoclonal antibodies Y2, BWR4, 400t $\mu 23$, or PL2-3 (in-house) were used as standards for the anti-Sm, anti-RNA, rheumatoid factor, and anti-nucleosome measurements respectively.

$q R T-P C R$. For assessing deletion efficiency of TLR9, qRT-PCR was performed on genomic DNA extracted from purified cells. The amount of TLR9 in each sample was normalized to the unaffected gene IL-10 or GAPDH. Primer sequences are as follows: TLR9 forward 5' ACTCCGACTTCGTCCACCT, reverse 5' GGCTCAATGGTCATGTGGCA; IL-10 forward 5' ATAACTGCACCCACTTCCCA, reverse 5' GTCCTGCATTAAGGAGTCGG; GAPDH forward 5' TCCCACTCTTCCACCTTCGA, reverse $5^{\prime}$ AGTTGGGATAGGGCCTCTCTT. qRT-PCR was performed with Agilent Brilliant II SYBR Green qPCR kit on a Stratagene Mx3000P or Roche LightCycler 96.

Ex vivo stimulation. To assess TLR9 functionality, splenocytes from the indicated mice as noted in individual figures were isolated as per flow cytometry methods, with the exception that all media was azide free. Splenic single-cell suspensions were sorted using a BD FACS Aria II. Isolated B cells were plated at a concentration of 250,000 cells per well in a 96-well plate. Cells were stimulated with the indicated concentration of CpG DNA, CpG ODN 1826 (Hokkaido System Science) for 72 hours. Supernatants were collected at 72 hours and used for anti-IgM ELISA as described (59).

Evaluation of clinical disease. Proteinuria was measured by Albustix strips (Bayer). Kidneys were formalin-fixed, paraffin-embedded, and H\&E-stained. Glomerular and interstitial nephritis were scored by a pathologist in a blinded manner. Kidneys were removed, bisected, formalin-fixed, paraffin-embedded, and H\&E-stained. Glomerulonephritis was scored on a scale of 1 to 6 (1, normal kidney; 2 , mesangial expansion and increased mesangial cellularity and patent capillary loops; 3, enlarged glomeruli with moderate endocapillary hypercellularity; 4, 3+ with marked endocapillary hypercellularity and loss of patency of most capillary loops; 5 , few glomeruli with necrosis [karyorrhexis] or few active [cellular or fibrocellular] or organized [fibrous] crescents; 6, many active [cellular or fibrocellular] or organized [fibrous] crescents, necrosis [karyorrhexis], obliteration of glomer- ular architecture, segmental /global sclerosis). Interstitial nephritis was scored on a scale of 1 to 4 in a blinded manner $(1$, minimal inflammation [lymphocytes and plasma cells] confined to the perivascular area; 2, expansion of inflammation throughout the interstitial space but maintained in discrete area; 3 , diffuse infiltrates in over $40 \%$ of high-powered fields; 4 , diffuse infiltrate throughout the entire interstitial space). Dermatitis was scored based on the extent of dermatitis on the dorsum of the neck and back. The macroscopic surface area was scored from 0 to 5 for an affected area up to $9.1 \mathrm{~cm}^{2}$, with up to 1 additional point for the presence of ear (a quarter point each) and muzzle (a half point) dermatitis.

Antibodies used for FACS staining. Antibodies used for FACS surface and intracellular staining were as follows: IA/E-PE (BioLegend, M5/114.15.2), Bst-2-biotin (in-house conjugated, 927), CD11c-PE/Cy7 (BD Pharmingen, HL3), CD45R-APC/Cy7 (BD Pharmingen, RA3-6B2), SiglecH-Al647 (eBioscience, eBio440c), CD19-Pacblue (in-house conjugated, 1D3.2), Ly6G-Al488 (in-house conjugated, 1A8), Gr1-PE/Cy7 (BioLegend, RB6-8C5), Gr1-PE (BioLegend, RB6-8C5), CD11b-APC/ Cy7 (BioLegend, M1/70), CD11b-PE (BioLegend, M1/70), F4/80Al647 (in-house conjugated, BM8), F4/80-APC (BioLegend, BM8), CD44-Al488 (in-house conjugated, 1M7), CD44-APC-Cy7 (BioLegend, 1M7), TcR $\beta$-APC/Cy7, (BioLegend, H57-597), TCRß-PE/Cy7, (BioLegend, H57-597), CD62L-PE/Cy7 (BioLegend, Mel-14), CD8-Al647 (in-house conjugated, TIB 105), CD4-PE (in-house conjugated, GK1.5), CD138-PE (BD Pharmingen, 281-2), CD19-AI647 (in-house conjugated, 1D3.2), kappa-Al488 (in-house conjugated, 187.1), and Ly6B.2-Fitc (AbD Serotec, 7/4). TLR9-PE (BD Pharmingen J15A7).

Mice. A conditional allele of TLR9 was generated using homologous recombination in ES cells by Ingenious Targeting Laboratory (Figure 2A). Mice carrying the TLR9 floxed allele (TLR9 ${ }^{\mathrm{fl} / \mathrm{f}}$ ) were crossed to MRL-MpJ-Fas ${ }^{\text {pr }} / \mathrm{J}$ (Jackson Laboratory) 10 times. CD11c-Cre (69) and CD19-Cre (70) mice were backcrossed to MRL-MpJ-Fas ${ }^{\mathrm{lpr}} / \mathrm{J}$ as previously described (32), and LysM-Cre (Jackson Laboratory) and MRP8-Cre (Jackson Laboratory) were backcrossed to MRL-MpJ-Fas ${ }^{\mathrm{lpr}} / \mathrm{J}$ at least 9 generations. Experimental cohorts were generated by intercrossing individual promoter-driven $\mathrm{Cre}^{+} \mathrm{TLR}^{\mathrm{fl} / \mathrm{fl}}$ with TLR9 ${ }^{\mathrm{fl} / \mathrm{fl}} \mathrm{MRL}-$ $\mathrm{MpJ} \mathrm{Fas}^{\mathrm{pr}} / \mathrm{J}$ mice and were aged to the indicated time points. To generate mice conditionally overexpressing TLR9, HA-tagged TLR9 was knocked into the Rosa26 locus (R26 FL ST TLR9-HA ${ }^{+/+}$referred to as $\left.R o s a^{\text {Trr }}\right)$ as described in Figure 6 and ref. 71, and were backcrossed to MRL-MpJ-Fas ${ }^{\mathrm{pr}} / \mathrm{J}$ for 7 generations. Resulting offspring were crossed to CD19-Cre MRL/lpr mice to generate the experimental cohort. To delineate the effect of B cell overexpression of TLR9 in lupus disease, R26 FL ST TLR9 $\mathrm{HA}^{+/+}$mice were also crossed to B6.Fc $\gamma \mathrm{RIIB}^{-/}$.Yaa mice (a gift from Silvia Bolland NIAD, NIH, Rockville, Maryland, USA) and the resulting progeny crossed to CD19-Cre mice to generate the experimental cohort. Disease pathology was evaluated in male mice at 24 weeks of age. BALB/c mice controls were purchased from Jackson Laboratory and TLR9 ${ }^{-/}$of mixed genetic background (72) were bred to $\mathrm{BALB} / \mathrm{c}$ in our colony.

Statistics. Statistics were calculated in GraphPad Prism by 1- or 2-tailed Mann-Whitney $U$ test or Student's $t$ test, and $\chi^{2}$ tests were used as indicated throughout. $P$ values are represented as ${ }^{*} P<0.05,{ }^{* *} P<$ $0.01,{ }^{* *} P<0.001,{ }^{* * *} P<0.0001$.

Study approval. All work was approved by the Institutional Animal Care and Use Committees of the University of Pittsburgh and Yale University. 


\section{Author contributions}

SJ, RAG, JST, and MJS conceived the project and designed experiments. SJ designed and validated the Tlr $9^{f l}$ and Ros $a^{\text {Tlr }}$ constructs. JST analyzed the flow data. SJ and RAG analyzed the qPCR data. KMN and RAG assisted with data interpretation and experimental design. CL optimized TLR9 expression via flow cytometry (Figure 1) and helped with data interpretation. JST, SJ, and MJS wrote the manuscript. MK and SB conducted pathologic analysis of the kidney tissue. The order of the 3 co-first authors was determined as follows: JST was responsible for the majority of the writing of the manuscript and completed the final analysis as well as the initial experiments on one cohort, and thus was listed first; SJ designed and made the $T l r 9^{f l}$ and $R o s a^{T r}$ constructs used for the majority of the experiments and completed initial analysis of 3 of the cohorts, so she was listed second; RG completed the majority of the experiments and initial analysis for 2 of the cohorts, so was listed third.

\section{Acknowledgments}

We would like to acknowledge significant contributions for technical work provided by Tony Marinov, Brady Marburger, Minjung Katie Kim, Yujuan Wang, and Lino Teichmann to help complete this work. We would also like to recognize the assistance the Flow Cytometry Core and the excellent animal care provided by the Department of Laboratory Animal Research. This research was supported by R37AI118841 (to MJS) and 2P01AR050256 (to MJS and Ann Marshak-Rothstein). JST was funded by NIH 25 grant 2T32AI0189443-06 and NIH Career Development Awards 5KL2TR001856-02 and 1K08AR075056-01.

Address correspondence to: Mark Shlomchik, W1052 Biomedical Science Tower, 200 Lothrop Street, Pittsburgh, Pennsylvania 15261, USA. Phone: 412.648.8771; Email: mshlomch@pitt.edu.
1. Marshak-Rothstein A. Toll-like receptors in systemic autoimmune disease. Nat Rev Immunol. 2006;6(11):823-835.

2. Richez C, Blanco P, Rifkin I, Moreau JF, Schaeverbeke T. Role for toll-like receptors in autoimmune disease: the example of systemic lupus erythematosus. Joint Bone Spine. 2011;78(2):124-130.

3. Song GG, Kim JH, Seo YH, Choi SJ, Ji JD, Lee YH. Associations between interleukin 1 polymorphisms and susceptibility to systemic lupus erythematosus: a meta-analysis. Hum Immunol. 2014;75(1):105-112.

4. Zhao R, Zhou H, Su SB. A critical role for interleukin-1 $\beta$ in the progression of autoimmune diseases. Int Immunopharmacol. 2013;17(3):658-669.

5. Lee YH, Choi SJ, Ji JD, Song GG. Association between toll-like receptor polymorphisms and systemic lupus erythematosus: a meta-analysis update. Lupus. 2016;25(6):593-601.

6. Celhar T, Magalhães R, Fairhurst AM. TLR7 and TLR9 in SLE: when sensing self goes wrong. Immunol Res. 2012;53(1-3):58-77.

7. Nickerson KM, et al. TLR9 regulates TLR7- and MyD88-dependent autoantibody production and disease in a murine model of lupus. J Immunol. 2010;184(4):1840-1848.

8. Christensen SR, Shupe J, Nickerson K, Kashgarian M, Flavell RA, Shlomchik MJ. Toll-like receptor 7 and TLR9 dictate autoantibody specificity and have opposing inflammatory and regulatory roles in a murine model of lupus. Immunity. 2006;25(3):417-428.

9. Fairhurst AM, et al. Yaa autoimmune phenotypes are conferred by overexpression of TLR7. Eur J Immunol. 2008;38(7):1971-1978.

10. Mohammad Hosseini A, Majidi J, Baradaran B, Yousefi M. Toll-like receptors in the pathogenesis of autoimmune diseases. Adv Pharm Bull. 2015;5(Suppl 1):605-614.

11. Nickerson KM, Wang Y, Bastacky S, Shlomchik MJ. Toll-like receptor 9 suppresses lupus disease in Fas-sufficient MRL Mice. PLOS ONE. 2017;12(3):e0173471.

12. Bossaller L, et al. TLR9 deficiency leads to accelerated renal disease and myeloid lineage abnormalities in pristane-induced murine lupus. J Immunol. 2016;197(4):1044-1053.
13. Jackson SW, et al. Opposing impact of B cellintrinsic TLR7 and TLR9 signals on autoantibody repertoire and systemic inflammation. J Immunol. 2014;192(10):4525-4532.

14. Lartigue A, et al. Role of TLR9 in anti-nucleosome and anti-DNA antibody production in lpr mutation-induced murine lupus. J Immunol. 2006;177(2):1349-1354.

15. Santiago-Raber ML, et al. Critical role of TLR7 in the acceleration of systemic lupus erythematosus in TLR9-deficient mice. J Autoimmun. 2010;34(4):339-348.

16. Stoehr AD, et al. TLR9 in peritoneal B-1b cells is essential for production of protective self-reactive IgM to control Th17 cells and severe autoimmunity. J Immunol. 2011;187(6):2953-2965.

17. Yu P, et al. Toll-like receptor 9-independent aggravation of glomerulonephritis in a novel model of SLE. Int Immunol. 2006;18(8):1211-1219.

18. Sang A, Zheng YY, Morel L. Contributions of $\mathrm{B}$ cells to lupus pathogenesis. Mol Immunol. 2014;62(2):329-338.

19. Chan OT, Madaio MP, Shlomchik MJ. B cells are required for lupus nephritis in the polygenic, Fas-intact MRL model of systemic autoimmunity. JImmunol. 1999;163(7):3592-3596.

20. Kansal R, et al. Sustained B cell depletion by CD19-targeted CAR T cells is a highly effective treatment for murine lupus. Sci Transl Med. 2019;11(482):eaav1648.

21. Kaplan MJ. Neutrophils in the pathogenesis and manifestations of SLE. Nat Rev Rheumatol. 2011;7(12):691-699.

22. Miyake K, Onji M. Endocytosis-free DNA sensing by cell surface TLR9 in neutrophils: rapid defense with autoimmune risks. Eur J Immunol. 2013;43(8):2006-2009.

23. Tas SW, Quartier P, Botto M, Fossati-Jimack L. Macrophages from patients with SLE and rheumatoid arthritis have defective adhesion in vitro, while only SLE macrophages have impaired uptake of apoptotic cells. Ann Rheum Dis. 2006;65(2):216-221.

24. Li Y, Lee PY, Reeves WH. Monocyte and macrophage abnormalities in systemic lupus erythematosus. Arch Immunol Ther Exp (Warsz). 2010;58(5):355-364.
25. Teichmann LL, Ols ML, Kashgarian M, Reizis B, Kaplan DH, Shlomchik MJ. Dendritic cells in lupus are not required for activation of $\mathrm{T}$ and $\mathrm{B}$ cells but promote their expansion, resulting in tissue damage. Immunity. 2010;33(6):967-978.

26. Klarquist J, Zhou Z, Shen N, Janssen EM. Dendritic cells in systemic lupus erythematosus: from pathogenic players to therapeutic tools. Mediators Inflamm. 2016;2016:5045248.

27. Kim JM, Park SH, Kim HY, Kwok SK. A plasmacytoid dendritic cells-type I interferon axis is critically implicated in the pathogenesis of systemic lupus erythematosus. Int J Mol Sci. 2015;16(6):14158-14170.

28. Onji M, et al. An essential role for the $\mathrm{N}$-terminal fragment of Toll-like receptor 9 in DNA sensing. Nat Commun. 2013;4:1949.

29. Heng TS, Painter MW, Immunological Genome Project Consortium. The Immunological Genome Project: networks of gene expression in immune cells. Nat Immunol. 2008;9(10):1091-1094.

30. Lindau D, et al. Primary blood neutrophils express a functional cell surface Toll-like receptor 9. Eur JImmunol. 2013;43(8):2101-2113.

31. El-Sayed OM, et al. Intact Toll-like receptor 9 signaling in neutrophils modulates normal thrombogenesis in mice. J Vasc Surg. 2016;64(5):1450-1458.e1.

32. Teichmann LL, Schenten D, Medzhitov R, Kashgarian M, Shlomchik MJ. Signals via the adaptor MyD88 in B cells and DCs make distinct and synergistic contributions to immune activation and tissue damage in lupus. Immunity. 2013;38(3):528-540.

33. Abram CL, Roberge GL, Hu Y, Lowell CA. Comparative analysis of the efficiency and specificity of myeloid-Cre deleting strains using ROSA-EYFP reporter mice. J Immunol Methods. 2014;408:89-100.

34. Hayashi F, Means TK, Luster AD. Toll-like receptors stimulate human neutrophil function. Blood. 2003;102(7):2660-2669.

35. Meng X, et al. Protective role of surface Toll-like receptor 9 expressing neutrophils in local inflammation during systemic inflammatory response syndrome in mice. Mol Immunol. 2017;90:74-86. 36. Merkenschlager J, et al. MHC class II cell-auton- 
omously regulates self-renewal and differentiation of normal and malignant B cells. Blood. 2019;133(10):1108-1118.

37. Nündel K, et al. Cell-intrinsic expression of TLR9 in autoreactive B cells constrains BCR/ TLR7-dependent responses. J Immunol. 2015;194(6):2504-2512.

38. Giles JR, Kashgarian M, Koni PA, Shlomchik MJ. B cell-specific MHC class II deletion reveals multiple nonredundant roles for B Cell Antigen Presentation in Murine Lupus. JImmunol. 2015;195(6):2571-2579.

39. Bolland S, Yim YS, Tus K, Wakeland EK, Ravetch JV. Genetic modifiers of systemic lupus erythematosus in FcgammaRIIB(-/-) mice. J Exp Med. 2002;195(9):1167-1174.

40. Devarapu SK, Anders HJ. Toll-like receptors in lupus nephritis. J Biomed Sci. 2018;25(1):35.

41. Conti F, et al. Kidney expression of Toll like receptors in lupus nephritis: quantification and clinicopathological correlations. Mediators Inflamm. 2016;2016:7697592.

42. Liu J, Willet SG, Bankaitis ED, Xu Y, Wright $\mathrm{CV}, \mathrm{Gu}$ G. Non-parallel recombination limits Cre-LoxP-based reporters as precise indicators of conditional genetic manipulation. Genesis. 2013;51(6):436-442.

43. Han SJ, Li H, Kim M, Shlomchik MJ, Lee HT. Kidney proximal tubular TLR9 exacerbates ischemic acute kidney injury. J Immunol. 2018;201(3):1073-1085.

44. Deane JA, et al. Control of toll-like receptor 7 expression is essential to restrict autoimmunity and dendritic cell proliferation. Immunity. 2007;27(5):801-810.

45. Celhar T, et al. RNA sensing by conventional dendritic cells is central to the development of lupus nephritis. Proc Natl Acad Sci USA. 2015;112(45):E6195-E6204.

46. Sindhava VJ, et al. A TLR9-dependent checkpoint governs B cell responses to DNA-containing antigens. JClin Invest. 2017;127(5):1651-1663.

47. Nickerson KM, Christensen SR, Cullen JL, Meng W, Luning Prak ET, Shlomchik MJ. TLR9 promotes tolerance by restricting survival of anergic anti-DNA B cells, yet is also required for their activation. J Immunol. 2013;190(4):1447-1456.

48. Fukui R, et al. Unc93B1 restricts systemic lethal inflammation by orchestrating Toll-like receptor 7 and 9 trafficking. Immunity. 2011;35(1):69-81.

49. Fukui R, et al. Unc93B1 biases Toll-like receptor responses to nucleic acid in dendritic cells toward DNA- but against RNA-sensing. J Exp Med. 2009;206(6):1339-1350.

50. Brinkmann MM, Spooner E, Hoebe K, Beutler B, Ploegh HL, Kim YM. The interaction between the ER membrane protein UNC93B and TLR3, 7, and 9 is crucial for TLR signaling. J Cell Biol. 2007;177(2):265-275.

51. Lee BL, et al. UNC93B1 mediates differential trafficking of endosomal TLRs. Elife. 2013;2:e00291.

52. Kim YM, Brinkmann MM, Paquet ME, Ploegh HL. UNC93B1 delivers nucleotide-sensing toll-like receptors to endolysosomes. Nature. 2008;452(7184):234-238.

53. Avalos AM, et al. Cell-specific TLR9 trafficking in primary APCs of transgenic TLR9-GFP mice. JImmunol. 2013;190(2):695-702.

54. Honda K, et al. Spatiotemporal regulation of MyD88-IRF-7 signalling for robust type-I interferon induction. Nature. 2005;434(7036):1035-1040

55. Park B, Brinkmann MM, Spooner E, Lee CC, Kim YM, Ploegh HL. Proteolytic cleavage in an endolysosomal compartment is required for activation of Toll-like receptor 9. Nat Immunol. 2008;9(12):1407-1414.

56. Bai Y, Tong Y, Liu Y, Hu H. Self-dsDNA in the pathogenesis of systemic lupus erythematosus. Clin Exp Immunol. 2018;191(1):1-10.

57. Mummert E, Fritzler MJ, Sjöwall C, Bentow C, Mahler M. The clinical utility of anti-doublestranded DNA antibodies and the challenges of their determination. J Immunol Methods. 2018;459:11-19.

58. Green NM, Marshak-Rothstein A. Toll-like receptor driven $B$ cell activation in the induction of systemic autoimmunity. Semin Immunol. 2011;23(2):106-112.

59. Chan OT, Hannum LG, Haberman AM, Madaio MP, Shlomchik MJ. A novel mouse with B cells but lacking serum antibody reveals an antibody-independent role for B cells in murine lupus. JExp Med.1999;189(10):1639-1648.

60. Witte T, et al. IgM anti-dsDNA antibodies in systemic lupus erythematosus: negative association with nephritis. SLE Study Group. Rheumatol Int. 1998;18(3):85-91.

61. Diaz M. The role of activation-induced deaminase in lupus nephritis. Autoimmunity. 2013;46(2):115-120.

62. Keiserman B, Ronchetti MR, Monticielo OA, Keiserman MW, Staub HL. Concomitance of IgM and IgG anti-dsDNA antibodies does not appear to associate to active lupus nephritis. Open Rheumatol J. 2013;7:101-104

63. Gies V, et al. Impaired TLR9 responses in B cells from patients with systemic lupus erythematosus. JCI Insight. 2018;3(5):96795.

64. Sieber J, et al. Active systemic lupus erythematosus is associated with a reduced cytokine production by B cells in response to TLR9 stimulation. Arthritis Res Ther. 2014;16(6):477.

65. Schmoll HJ, et al. Maintenance treatment with the immunomodulator MGN1703, a Toll-like receptor 9 (TLR9) agonist, in patients with metastatic colorectal carcinoma and disease control after chemotherapy: a randomised, double-blind, placebo-controlled trial. J Cancer Res Clin Oncol. 2014;140(9):1615-1624.

66. Iribarren K, et al. Trial Watch: Immunostimulation with Toll-like receptor agonists in cancer therapy. Oncoimmunology. 2016;5(3):e1088631.

67. Campbell AM, Kashgarian M, Shlomchik MJ. NADPH oxidase inhibits the pathogenesis of systemic lupus erythematosus. Sci Transl Med. 2012;4(157):157ra141.

68. Gordon RA, et al. Lupus and proliferative nephritis are PAD4 independent in murine models. JCI Insight. 2017;2(10):92926.

69. Caton ML, Smith-Raska MR, Reizis B. NotchRBP-J signaling controls the homeostasis of CD8- dendritic cells in the spleen. J Exp Med. 2007;204(7):1653-1664.

70. Rickert RC, Roes J, Rajewsky K. B lymphocytespecific, Cre-mediated mutagenesis in mice. Nucleic Acids Res. 1997;25(6):1317-1318.

71. Roberts AW, Lee BL, Deguine J, John S, Shlomchik MJ, Barton GM. Tissue-resident macrophages are locally programmed for silent clearance of apoptotic cells. Immunity. 2017;47(5):913-927.e6.

72. Hemmi H, et al. A Toll-like receptor recognizes bacterial DNA. Nature. 2000;408(6813):740-745. 